\title{
Decision Support Technique Based on Neutrosophic Yager Aggregation Operators: Application in Solar Power Plant Locations-Case Study of Bahawalpur, Pakistan
}

\author{
Shabeer Khan, ${ }^{1}$ Saleem Abdullah, ${ }^{1}$ Shahzaib Ashraf $\mathbb{D D}^{2}{ }^{2}$ Ronnason Chinram $\left(\mathbb{D},{ }^{3}\right.$ \\ and Samruam Baupradist ${ }^{4}$ \\ ${ }^{1}$ Department of Mathematics, Abdul Wali Khan University, Mardan 23200, Pakistan \\ ${ }^{2}$ Department of Mathematics and Statistics, Bacha Khan University, Charsadda, Khyber PakhtunKhwa, Pakistan \\ ${ }^{3}$ Algebra and Applications Research Unit, Division of Computational Science, Faculty of Science, Prince of Songkla University, \\ Hat Yai, Songkhla 90110, Thailand \\ ${ }^{4}$ Department of Mathematics and Computer Science, Faculty of Science, Chulalongkorn University, Bangkok 10330, Thailand
}

Correspondence should be addressed to Ronnason Chinram; ronnason.c@psu.ac.th

Received 9 October 2020; Revised 5 November 2020; Accepted 13 November 2020; Published 2 December 2020

Academic Editor: Mingwei Lin

Copyright $\odot 2020$ Shabeer Khan et al. This is an open access article distributed under the Creative Commons Attribution License, which permits unrestricted use, distribution, and reproduction in any medium, provided the original work is properly cited.

\begin{abstract}
The problem of energy crisis and environmental pollution has been mitigated by the generation and use of solar power; however, the choice of locations for solar power plants is a difficult task because the decision-making process includes political, socioeconomic, and environmental aspects. Thus, several adverse consequences have been created by the choice of suboptimal locations. The objective of this paper is to address the integrated qualitative and quantitative multicriteria decision-making framework for the selection of solar power plant locations. Neutrosophic sets (NSs) are the latest extension of the ordinary fuzzy sets. The main characteristic of the neutrosophic sets is satisfying the condition that the sum of the truth, indeterminacy, and falsity grades must be at least zero and at most three. In this research, we establish novel operational laws based on the Yager $\mathrm{t}$-norm and t-conorm under neutrosophic environments (NE). Furthermore, based on these Yager operational laws, we develop a list of novel aggregation operators under NE. In addition, we design an algorithm to tackle the uncertainty to investigating the best solar power plant selection in five potential locations in Pakistan. A numerical example of solar power plant location problem is considered to show the supremacy and effectiveness of the proposed study. Also, a detailed comparison is constructed to evaluate the performance and validity of the established technique.
\end{abstract}

\section{Introduction}

Decision-making (DM) is one of the most common and frequent human activities aimed at selecting the best option with respect to a list of attributes. Due to the high capability of DM to model uncertain data, it has been extensively studied and applied successfully to economics, management, and other areas in recent years. Using fuzzy set theory to tackle the DM problems has become a hotspot in recent years because of the uncertainty in decision information. To handle fuzziness and vagueness information, Zadeh [1] introduced the fuzzy sets (FSs) by using only membership degree in $[0,1]$, and then Atanassov [2] proposed the intuitionistic FSs by using both positive and negative membership grades in $[0,1]$. Many extensions of ordinary FSs have been introduced by many researchers [3-13]. These modifications have often been used in the development of DM issues in an uncertain environment.

Although intuitionistic FS can deal with incomplete and uncertainty information, it cannot handle inconsistent information better in real situations, for example, Son [14]; in the election of village director, the voting results can be divided into three categories: "vote for," "neutral voting," and "vote against." "Neutral voting" means that the voting paper is a white paper rejecting both agree and disagree for the candidate but still takes the vote. This example has 
happened in reality, but intuitionistic FS could not handle it. In order to solve these problems, Cuong $[15,16]$ proposed picture FS, which contains three aspects of information: yes, neutral, and no. It can deal with inconsistent information. Up to now, many outstanding contributions have been made in the research of picture FSs, for example, Wei [17] introduced the some novel AgOs for Picture FS and discussed their applications in DM problems. Ashraf et al. [18] highlights the deficiency in the existing operational laws and established novel improved AgOs to tackle the uncertainty in complex real-life DM problems under picture fuzzy environment. Khan et al. [19] established the novel extension, generalized picture fuzzy soft sets, and discussed their DM applications. Khan et al. [20] established the novel AgOs using logarithmic function and algebraic norm under picture fuzzy environment. Qiyas et al. [21] presented the linguistic information and algebraic norm-based novel AgOs using picture FSs. Ashraf et al. [22] presented the cleaner production evaluation technique based on the cubic picture fuzzy AgOs using distance information measures. Qiyas et al. [23] utilized linguistic variables to develop the list of AgOs based on Dombi operational laws to tackle the DM problems of real world. Ashraf and Abdullah [24] introduced algebraic norm-based AgOs under cubic picture FS and discussed their applications in the decision problem.

Picture FS is an important generalization of FS theory, but picture FS would be meaningless in certain DM problems with the constant complexities of human information. Ashraf et al. [25, 26] introduced a new and more general concept spherical fuzzy set (spherical FS), which is an extension of FS by further slackening the condition that $0 \leq b^{2}(x)+I^{2}(x)+\partial^{2}(x) \leq 1$. It should also be noted that the acceptable spherical framework gives experts more opportunity to express their belief in supporting membership, although, spherical FS have been successfully applied in some fields, especially in decision-making fields.

As aggregation operators (AgOs) make a massive contribution to the integration of DM issues, numerous studies have examined very valuable contributions to the incorporation of spherical FS AgOs. Ashraf et al. [26] established spherical AgO-based algebraic norm to tackle inaccurate data in DM problems, in [27], presented the spherical FS norms representation under SF settings. Jin et al. [28] developed the linguistic function-based SF AgOs and, in [29], presented the list of SF Dombi AgOs using Dombi norm. GRA methodology based on spherical linguistic fuzzy Choquet integral is proposed [30] for SF information. Rafiq et al. [31] developed the cosine function-based novel similarity measures, and Ashraf et al. [32] developed the distance measure-based AgOs to tackle the inaccurate data in DM. Zeng et al. [33] introduced TOPSIS methodology under SF rough sets. Gündoğdu and Kahraman [34] established the TOPSIS methodology under spherical FSs and also proposed their applications. Ashraf and Abdullah [35] presented the emergency decision-making technique of coronavirus using the spherical FSs. Ashraf et al. [36] introduced the symmetric sum-based AgOs under spherical FSs to tackle the uncertainty in daily life DM problems. Gundogdu and Kahraman [37] established the generalized methodology based on
WASPAS under spherical FSs. Jin et al. [38] utilized the logarithmic function to developed the novel SF AgOs under spherical FSs. Shishavan et al. [39] established the list of similarity measures to tackle the uncertainty in the form of spherical fuzzy environment. Gündoğdu and Kahraman [40] presented the new AHP technique to tackle the uncertainty in renewable energy and, in [41], discussed the spherical fuzzy QFD technique to tackle the uncertainty in robot technology development problems.

While the presentation of fuzzy sets and their extended sets provides more decision-making space, there are still some restrictions. For instance, it is impossible to solve the discontinuity and inconsistency of data so that the NS emerge as the times require. For the very first time, the notion of three parameters is taken into account, namely, the degree of truth, indeterminacy, and falsity. This theory can help decision makers to express their views more precisely and in detail and to address problems that the fuzzy set cannot resolve. The concept of neutrosophic sets was first proposed by Smarandache [42]. It is a philosophical branch and is a mathematical model to understand not only the origin, nature, and scope of neutrality, but also the interaction between their various conceptual ranges. Such improvements have been made to improve capability in order to address DM issues in ambiguous environments. Many authors contribute to NS theory to tackle the uncertain data in DM problems, such as Ye [43] established the DM approach based on AgOs under NSs, Peng et al. [44] presented the power AgOs for NSs and discussed their applicability in DM issues. Chen and Ye [45] established the Dombi normbased novel AgOs under SVNNs, Liu et al. [46] introduced Hamacher norm-based generalized AgOs to tackle the uncertain data in the form of neutrosophic numbers, Wei and Zhang [47] presented the Bonferroni mean-based power AgOs for single valued NSs to addressed the multiple attribute DM problems, Liu et al. [48] established the group DM methodology based on Heronian mean power AgOs under linguistic NS information to address the uncertain and inaccurate data in DM problems, and Garg [49] established the hybrid methodology with linguistic variables and single-valued NS-based prioritized AgOs and discussed their applicability to address the uncertain data in DM problems.

It is evident that the abovementioned AgOs are focused on the algebraic, Einstein, Dombi, and Hamacher norms under single-valued NSs for the implementation of the combination process. Algebraic, Einstein, Dombi, and Hamacher product and sum are not fundamental singlevalued NSs operations that describe the union and the intersection of any two single-valued NSs. A general union and intersection under NS information can be developed from a generalized norm, i.e., instances of deferent-norm families may be used to execute the respective intersections and unions under single-valued NSs environment. The Yager product and sum are good replacement of the algebraic, Einstein, Dombi, and Hamacher product for an intersection and union and is capable of delivering smooth estimates of the algebraic product and sum. However, there seems to be little work in the literature on aggregation approaches that 
use the Yager operations on FS theory to aggregate the fuzzy numbers. Akram and Shahzadi [50] introduced the q-rung orthopair FS-based Yager AgOs to tackle the DM problems. Akram et al. [51] presented the Yager norm-based AgOs under complex Pythagorean FSs and discussed their application in DM problems. Shahzadi et al. [52] presented the DM approach based on Yager operational laws under Pythagorean information. Garg et al. [53] presented the DM problem of COVID-19 Testing Facility using Fermatean FS and Yager norm information.

From the above analysis, we note that, in many practical applications, various aggregation operators have been put forward and implemented, although, in practical problems, many existing AgOs are not capable to address such specific cases. In some circumstances, many of these may result in unreasonable or counterintuitive results. Certain new regulations built without a simple function may have a complicated description. However, generalized aggregation operators for SVNSs continue to be an open subject that attracts many researchers. Therefore, in this article, our aim is to present some novel single-valued neutrosophic Yager operational law-based Yager AgOs to tackle the uncertainty in DM real-world problems with a more effective and efficient way. The contribution and originality of this study are summarized as follows:

(i) Novel ranking methodology and Yager norm-based novel operational laws for single-valued NSs are proposed

(ii) The new Yager averaging/geometric aggregation operators are proposed to aggregate the uncertainties in the form of single-valued NS environment

(iii) Decision-making algorithm is proposed to tackle the DM real-world problems

(iv) A real-life numerical application about solar power plant location selection problem in Pakistan is discussed to show the applicability of the proposed technique

The rest of this article shall be organized as set out below. Section 2 provides basic information concerning single-valued NSs. Section 3 describes the Yager operations of single-valued NSs. Section 4, presented as the cornerstone of this work, proposes novel neutrosophic Yager AgOs based on the Yager norm, together with the associated proof of its properties. Section 5 introduces the novel methodology for interacting with the ambiguity in DM problems in order to pick the best alternative according to the list of attributes. Section 6 provides a numerical application about solar power plant location selection problem which is used to illustrate the designed MAGDM method, and a comparative analysis with some existing frameworks to MAGDM is discussed in Section 7. The article is concluded in Section 8.

\section{Preliminaries}

The section provides some basic information on the followup criteria for the short-term tasks' Fuzzy set theory, spherical FS theory, and single-valued NS theory.

Definition 1 (see [1]). Let $U$ be the given collection, and a fuzzy set (FS) $\mathscr{F}$ in $U$ having one function is

$$
\mathscr{F}=\{\langle x, b(x)\rangle \mid x \in U\},
$$

where $b: U \longrightarrow[0,1]$ representing the positive grade of membership of $\mathscr{F}$ in $U$.

Definition 2 (see [2]). Let $U$ be the given collection, and an intuitionistic FS $\mathscr{F}_{i}$ in $U$ having two functions is

$$
\mathscr{F}_{i}=\{\langle x, b(x), \partial(x)\rangle \mid x \in U\},
$$

where $b, \partial: U \longrightarrow[0,1]$ representing the positive and negative grades of membership of $\mathscr{F}$ in $U$, such that $\forall x \in U$, $0 \leq b(x)+\partial(x) \leq 1$.

Definition 3 (see [15]). Let $U$ be the given collection, and a picture FS $\mathscr{F}_{p}$ in $U$ having three functions is

$$
\mathscr{F}_{p}=\{\langle x, b(x), I(x), \partial(x)\rangle \mid x \in U\},
$$

where $b, I$, and $\partial: U \longrightarrow[0,1]$ representing the positive, neutral, and negative grades of membership of $\mathscr{F}$ in $U$, such that $\forall x \in U, 0 \leq b(x)+I(x)+\partial(x) \leq 1$.

Definition 4 (see $[25,26])$. Let $U$ be the given collection, a spherical FS $\mathscr{F}_{s}$ in $U$ having three functions is

$$
\mathscr{F}_{s}=\{\langle x, b(x), I(x), \partial(x)\rangle \mid x \in U\},
$$

where $b, I$, and $\partial: U \longrightarrow[0,1]$ representing the positive, neutral, and negative grades of membership of $\mathscr{F}$ in $U$, such that $\forall x \in U, 0 \leq b^{2}(x)+I^{2}(x)+\partial^{2}(x) \leq 1$.

Definition 5 (see [42]). Let $U$ be the given collection, and a neutrosophic set (NS) $\mathscr{F}_{n}$ in $U$ having three functions is

$$
\mathscr{F}_{n}=\{\langle x, b(x), I(x), \partial(x)\rangle \mid x \in U\},
$$

where $b, I$, and $\partial: U \longrightarrow] 0^{-}, 1^{+}[$representing the truth, indeterminacy and falsity grades of membership of $\mathscr{F}$ in $U$, such that $\forall x \in U, 0^{-} \leq b(x)+I(x)+\partial(x) \leq 3^{+}$.

Definition 6 (see [54]). Let $U$ be the given collection, and a single-valued NS $\mathscr{F}_{n}$ in $U$ having three functions is

$$
\mathscr{F}_{n}=\{\langle x, b(x), I(x), \partial(x)\rangle \mid x \in U\},
$$


where $b, I$, and $\partial: U \longrightarrow[0,1]$ representing the truth, indeterminacy, and falsity grades of membership of $\mathscr{F}$ in $U$, such that $\forall x \in U, 0 \leq b(x)+I(x)+\partial(x) \leq 3$.

We represent $\operatorname{SVNS}(U)$ the collection of single-valued NSs. Wang et al. [54], Ye [55], and Zhang and Bo [56] developed the initial operating rules which are discussed as follows.

Definition 7 (see [56]). Let $\mathscr{F}_{n t_{(1)}}, \mathscr{F}_{n t_{(2)}} \in \operatorname{SVNS}(U)$; then,

(1) $\mathscr{F}_{n t_{(1)}} \subseteq \mathscr{F}_{n t}$ iff $b_{1} \leq b_{2}, I_{1} \geq I_{2}$ and $\partial_{1} \geq \partial_{2}$. Clearly, $\mathscr{F}_{n t_{(1)}}=\mathscr{F}_{n t_{(2)}}$ if $\mathscr{F}_{n t_{(1)}} \subseteq \mathscr{F}_{n t_{(2)}}$ and $\mathscr{F}_{n t_{(2)}} \subseteq \mathscr{F}_{n t_{(1)}}$.

(2) $\mathscr{F}_{n t_{(1)}} \cap \mathscr{F}_{n t}=\left\{\inf \left(b_{1}, b_{2}\right), \sup \left(I_{1}, I_{2}\right), \sup \left(\partial_{1}, \partial_{2}\right)\right\}$.

(3) $\mathscr{F}_{n t_{(1)}} \cup \mathscr{F}_{n t_{(2)}}=\left\{\sup \left(b_{1}, b_{2}\right), \inf \left(I_{1}, I_{2}\right), \inf \left(\partial_{1}, \partial_{2}\right)\right\}$.

(4) $\mathscr{F}_{n t_{(1)}^{c}}^{c}=\left\{\partial_{1}, I_{1}, b_{1}\right\}$.
Definition 8 (see [54,57]). Let $\mathscr{F}_{n t_{(1)}}, \mathscr{F}_{n t_{(2)}} \in \operatorname{SVNS}(U)$; then, for $\varrho>0$,

(1) $\mathscr{F}_{n t_{(1)}} \otimes \mathscr{F}_{n t_{(2)}}=\left\{b_{1} b_{2}, I_{1}+I_{2}-I_{1} I_{2}, \partial_{1}+\partial_{2}-\partial_{1} \partial_{2}\right\}$

(2) $\mathscr{F}_{n t_{(1)}} \oplus \mathscr{F}_{n t_{(2)}}=\left\{b_{1}+b_{2}-b_{1} b_{2}, I_{1} I_{2}, \partial_{1} \partial_{2}\right\}$

(3) $\mathscr{F}_{n t_{(1)}}^{\mathrm{Q}}=\left\{b_{1}^{\mathrm{e}}, 1-\left(1-I_{1}\right)^{\varrho}, 1-\left(1-\partial_{1}\right)^{\varrho}\right\}$

(4) $\varrho \cdot \mathscr{F}_{n t_{(1)}}=\left\{1-\left(1-b_{1}\right)^{\varrho}, I_{1}^{\varrho}, \partial_{1}^{\varrho}\right\}$

Definition 9. Let $\quad \mathscr{F}_{h}=\left(b_{h}(x), I_{h}(x), \partial_{h}(x)\right) \in \mathrm{SVNN}$ $(U)(h \in \mathbb{N})$. Then, the weighted averaging AgOs for $\operatorname{SVNN}(U)$ is described as follows:

$$
\begin{aligned}
\operatorname{SVNWA}\left(\mathscr{F}_{1}, \mathscr{F}_{2}, \ldots, \mathscr{F}_{n}\right) & =\rho_{1} \mathscr{F}_{1} \oplus \rho_{2} \mathscr{F}_{2} \oplus \cdots \oplus \rho_{n} \mathscr{F}_{n}, \\
& =\sum_{h=1}^{n} \rho_{h} \mathscr{F}_{h}, \\
& =\left\{1-\prod_{h=1}^{n}\left(1-b_{h}\right)^{\rho_{h}}, \prod_{h=1}^{n}\left(I_{h}\right)^{\rho_{h}}, \prod_{h=1}^{n}\left(\partial_{h}\right)^{\rho_{h}}\right\},
\end{aligned}
$$

where the weights $\left(\rho_{1}, \rho_{2}, \ldots, \rho_{h}\right)$ of $\mathscr{F}_{h}$ have $\rho_{h} \geq 0$ and $\sum_{h=1}^{n} \rho_{h}=1$.
Definition 10. Let $\mathscr{F}_{h}=\left(b_{h}(x), I_{h}(x), \partial_{h}(x)\right) \in \mathrm{SVNN}$ $(U)(h \in \mathbb{N})$. Then, the weighted geometric AgOs for $\operatorname{SFNV}(U)$ is described as follows:

$$
\begin{aligned}
\operatorname{SVNWG}\left(\mathscr{F}_{1}, \mathscr{F}_{2}, \ldots, \mathscr{F}_{n}\right) & =\mathscr{F}_{1}^{\rho_{1}} \otimes \mathscr{F}_{2}^{\rho_{2}} \otimes \cdots \otimes \mathscr{F}_{n}^{\rho_{n}}, \\
& =\prod_{h=1}^{n}\left(\mathscr{F}_{h}\right)^{\rho_{h}}, \\
& =\left\{\prod_{h=1}^{n}\left(b_{h}\right)^{\rho_{h}}, 1-\prod_{h=1}^{n}\left(1-I_{h}^{2}\right)^{\rho_{h}}, 1-\prod_{h=1}^{n}\left(1-\partial_{h}\right)^{\rho_{h}}\right\},
\end{aligned}
$$

where the weights $\left(\rho_{1}, \rho_{2}, \ldots, \rho_{h}\right)$ of $\mathscr{F}_{h}$ have $\rho_{h} \geq 0$ and $\sum_{h=1}^{n} \rho_{h}=1$.

Definition 11. Let $\mathscr{F}_{n t_{(1)}}=\left(b_{1}, I_{1}, \partial_{1}\right)$ and $\mathscr{F}_{n t_{(2)}}=\left(b_{2}, I_{2}\right.$, $\left.\partial_{2}\right) \in \operatorname{SVNS}(U) . \mathcal{\delta}_{\gamma}\left(\mathscr{F}_{n t(1)}\right)=b_{1}-I_{1}-\partial_{1}$ and $\delta_{\gamma}^{(2)}\left(\mathscr{F}_{n t}\right)=$ $b_{2}-I_{2}-\partial_{2}$ are the score values of SVNNs. Also, $\mathscr{A}_{y}\left(\mathscr{F}_{n t_{(1)}}\right)=b_{1}+I_{1}+\partial_{1}$ and $\mathscr{A}_{y}\left(\mathscr{F}_{n t_{(2)}}\right)=b_{1}+I_{1}+\partial_{1}$ are the accuracy values of SVNNs. If

(a) $\mathcal{S}_{\gamma}\left(\mathscr{F}_{n t_{(1)}}\right)<\mathcal{S}_{\gamma}\left(\mathscr{F}_{n t_{(2)}}\right) \Rightarrow \mathscr{F}_{n t_{(1)}}<\mathscr{F}_{n t_{(2)}}$

(b) $\underset{\mathscr{F}_{y}\left(\mathscr{F}_{n t_{(1)}}\right)}{\left.\mathscr{F}_{\mathscr{F}_{n t}}\right)}=\mathcal{S}_{\gamma}\left(\mathscr{F}_{n t_{(2)}}\right), \mathscr{A}_{y}\left(\mathscr{F}_{n t_{(1)}}\right)<\mathscr{A}_{y}\left(\mathscr{F}_{n t_{(2)}}\right) \Rightarrow$

(c) $\mathcal{S}_{\gamma}\left(\mathscr{F}_{n t_{(1)}}\right)=\mathcal{S}_{\gamma}\left(\mathscr{F}_{n t_{(2)}}\right), \mathscr{A}_{y}\left(\mathscr{F}_{n t_{(1)}}\right)=\mathscr{A}_{y}\left(\mathscr{F}_{n t_{(2)}}\right) \Rightarrow$
$\mathscr{F}_{n t_{(1)}}=\mathscr{F}_{n t_{(2)}}$

\section{New Operating Laws for Single-Valued NS}

In integrating information into one form and addressing DM issues, aggregation operators (AgOs) play a vital role. Aggregation facilitates the establishment of a number of choices in a system or a collection of objects that have come together or have been brought together. In recent years, AgOs based on FSs and their different hybrid compositions have provided a great deal of attention and become interesting because they can quickly execute functional areas of various regions. In this section, we propose the Yager normbased novel operational laws for single-valued NSs.

Definition 12 (see [58]). Yager's norms for any $p$ and $c \in \mathbb{R}$ :

(1) $\breve{T}(p, c)=1-\min \left(1,\left((1-p)^{\delta}+(1-c)^{\delta}\right)^{1 / \delta}\right)$ 
(2) $\widehat{S}(p, c)=\min \left(1,\left(p^{\delta}-c^{\delta}\right)^{1 / \delta}\right), \delta \in(0, \infty)$

Definition 13. Let $\mathscr{F}_{n t_{(N)}}, \mathscr{F}_{n t_{(2)}} \in \operatorname{SVNS}(U)$ with $\varrho, \delta>0$. The Yager operating laws (YOLs) are $\mathscr{F}_{n t}=(b(x), I(x), \partial(x))$ :

(1) $\mathscr{F}_{n t_{(1)}} \otimes \mathscr{F}_{n t_{(2)}}=\left\{1-\min \left(1,\left(\left(1-b_{1}\right)^{\delta}+\left(1-b_{2}\right)^{\delta}\right)\right.\right.$ $\left.1 / \delta), \min \left(1,\left(I_{1}^{\delta}+I_{2}^{\delta}\right)^{1 / \delta}\right), \min \left(1,\left(\partial_{1}^{\delta}+\partial_{2}^{\delta}\right)^{1 / \delta}\right)\right\}$

(2) $\mathscr{F}_{n t_{(1)}} \oplus \mathscr{F}_{n t_{(8)}}=$

$\left\{\min \left(1,\left(b_{1}^{\delta}+b_{2}^{(g)}\right)^{1 / \delta}\right), 1-\min (1,((1\right.$

$\left.\left.\left.-I_{1}\right)^{\delta}+\left(1-I_{2}\right)^{\delta}\right)^{1 / \delta}\right), 1-\min \left(1,\left(\left(1-\partial_{1}\right)^{\delta}+(1-\right.\right.$

$\left.\left.\left.\left.\partial_{2}\right)^{\delta}\right)^{1 / \delta}\right)\right\}$

(3) $\mathscr{F}_{n t_{(1)}}^{\rho}=\left\{1-\min \left(1,\left(\varrho\left(1-b_{1}\right)^{\delta}\right)^{1 / \delta}\right), \min \left(1,\left(\varrho I_{1}^{\delta}\right)\right.\right.$ $\left.1 / \delta), \min \left(1,\left(\varrho \partial_{1}^{\delta}\right)^{1 / \delta}\right)\right\}$

(4) $\varrho \cdot \mathscr{F}_{n t}=\left\{\min \left(1,\left(\varrho b_{1}^{\delta}\right)^{1 / \delta}\right), 1-\min \left(1,\left(\varrho\left(1-I_{1}\right)\right.\right.\right.$ $\left.\left.\delta)^{1 / \delta}\right), 1-\min \left(1,\left(\varrho\left(1-\partial_{1}\right)^{\delta}\right)^{1 / \delta}\right)\right\}$
Theorem 1. Let $\mathscr{F}_{n t_{(1)}}, \mathscr{F}_{n t_{(2)}} \in \operatorname{SVNS}(U)$ with $\varrho_{1}, \varrho_{2}>0$. Then,

(1) $\mathscr{F}_{n t_{(1)}} \oplus \mathscr{F}_{n t t_{(2)}}=\mathscr{F}_{n t_{(2)}} \oplus \mathscr{F}_{n t_{(1)}}$

(2) $\mathscr{F}_{n t_{(1)}} \otimes \mathscr{F}_{n t}=\mathscr{F}_{n t_{(2)}} \otimes \mathscr{F}_{n t}$

(3) $\varrho\left(\mathscr{F}_{n t_{(1)}} \oplus \mathscr{F}_{n t_{(2)}}\right)=\varrho \mathscr{F}_{n t_{(1)}} \oplus \varrho \mathscr{F}_{n t_{(2)}}$

(4) $\left(\varrho_{1}+\varrho_{2}\right) \mathscr{F}_{n t_{(1)}}=\varrho_{1} \mathscr{F}_{n t_{(1)}} \oplus \varrho_{2} \mathscr{F}_{n t_{(1)}}$

(5) $\left(\mathscr{F}_{n t_{(1)}} \otimes \mathscr{F}_{n t_{(2)}}\right)^{\varrho}=\mathscr{F}_{n t_{(1)}}^{\mathrm{e}} \otimes \mathscr{F}_{n t_{(2)}}^{\mathrm{e}}$

(6) $\mathscr{F}_{n t_{(1)}}^{\mathrm{e}_{1}} \otimes \mathscr{F}_{n t_{(1)}}^{\mathrm{e}_{2}}=\mathscr{F}_{n t_{(1)}}^{\left(\mathrm{e}_{1}+\mathrm{e}_{2}\right)}$

Proof. For any $\mathscr{F}_{n t_{(1)}}, \mathscr{F}_{n t_{(2)}} \in \operatorname{SVNS}(U)$ with $\varrho_{1}, \varrho_{2}>0$, we have

$$
\begin{aligned}
& \mathscr{F}_{n t_{(1)}} \oplus \mathscr{F}_{n t_{(2)}}=\left\{\min \left(1,\left(b_{1}^{\delta}+b_{2}^{\delta}\right)^{1 / \delta}\right), 1-\min \left(1,\left(\left(1-I_{1}\right)^{\delta}+\left(1-I_{2}\right)^{\delta}\right)^{1 / \delta}\right), 1-\min \left(1,\left(\left(1-\partial_{1}\right)^{\delta}+\left(1-\partial_{2}\right)^{\delta}\right)^{1 / \delta}\right)\right\} \\
& =\left\{\min \left(1,\left(b_{2}^{\delta}+b_{1}^{\delta}\right)^{1 / \delta}\right), 1-\min \left(1,\left(\left(1-I_{2}\right)^{\delta}+\left(1-I_{1}\right)^{\delta}\right)^{1 / \delta}\right), 1-\min \left(1,\left(\left(1-\partial_{2}\right)^{\delta}+\left(1-\partial_{1}\right)^{\delta}\right)^{1 / \delta}\right)\right\} \\
& =\mathscr{F}_{n t_{(2)}} \oplus \mathscr{F}_{n t_{(1)}}, \\
& \mathscr{F}_{n t_{(1)}} \otimes \mathscr{F}_{n t_{(2)}}=\left\{1-\min \left(1,\left(\left(1-b_{1}\right)^{\delta}+\left(1-b_{2}\right)^{\delta}\right)^{1 / \delta}\right), \min \left(1,\left(I_{1}^{\delta}+I_{2}^{\delta}\right)^{1 / \delta}\right), \min \left(1,\left(\partial_{1}^{\delta}+\partial_{2}^{\delta}\right)^{1 / \delta}\right)\right\} \\
& =\left\{1-\min \left(1,\left(\left(1-b_{2}\right)^{\delta}+\left(1-b_{1}\right)^{\delta}\right)^{1 / \delta}\right), \min \left(1,\left(I_{2}^{\delta}+I_{1}^{\delta}\right)^{1 / \delta}\right), \min \left(1,\left(\partial_{2}^{\delta}+\partial_{1}^{\delta}\right)^{1 / \delta}\right)\right\} \\
& =\mathscr{F}_{n t_{(2)}} \otimes \mathscr{F}_{n t_{(1)}} \text {, } \\
& \varrho\left(\mathscr{F}_{n t_{(1)}} \oplus \mathscr{F}_{n t_{(2)}}\right)=\varrho \cdot\left\{\min \left(1,\left(b_{1}^{\delta}+b_{2}^{\delta}\right)^{1 / \delta}\right), 1-\min \left(1,\left(\left(1-I_{1}\right)^{\delta}+\left(1-I_{2}\right)^{\delta}\right)^{1 / \delta}\right), 1-\min \left(1,\left(\left(1-\partial_{1}\right)^{\delta}+\left(1-\partial_{2}\right)^{\delta}\right)^{1 / \delta}\right)\right\} \\
& =\left\{\min \left(1,\left(\varrho b_{1}^{\delta}+\varrho b_{2}^{\delta}\right)^{1 / \delta}\right), 1-\min \left(1,\left(\varrho\left(1-I_{1}\right)^{\delta}+\varrho\left(1-I_{2}\right)^{\delta}\right)^{1 / \delta}\right), 1\right. \\
& \left.-\min \left(1,\left(\varrho\left(1-\partial_{1}\right)^{\delta}+\varrho\left(1-\partial_{2}\right)^{\delta}\right)^{1 / \delta}\right)\right\} \text {, } \\
& \varrho \mathscr{F}_{n t_{(1)}} \oplus \varrho \mathscr{F}_{n t_{(2)}}=\left\{\min \left(1,\left(\varrho b_{1}^{\delta}\right)^{1 / \delta}\right), 1-\min \left(1,\left(\varrho\left(1-I_{1}\right)^{\delta}\right)^{1 / \delta}\right), 1-\min \left(1,\left(\varrho\left(1-\partial_{1}\right)^{\delta}\right)^{1 / \delta}\right)\right\} \oplus\left\{\min \left(1,\left(\varrho b_{2}^{\delta}\right)^{1 / \delta}\right), 1\right. \\
& \left.-\min \left(1,\left(\varrho\left(1-I_{2}\right)^{\delta}\right)^{1 / \delta}\right), 1-\min \left(1,\left(\varrho\left(1-\partial_{2}\right)^{\delta}\right)^{1 / \delta}\right)\right\} \\
& =\left\{\min \left(1,\left(\varrho b_{1}^{\delta}+\varrho b_{2}^{\delta}\right)^{1 / \delta}\right), 1-\min \left(1,\left(\varrho\left(1-I_{1}\right)^{\delta}+\varrho\left(1-I_{2}\right)^{\delta}\right)^{1 / \delta}\right), 1\right. \\
& \left.-\min \left(1,\left(\varrho\left(1-\partial_{1}\right)^{\delta}+\varrho\left(1-\partial_{2}\right)^{\delta}\right)^{1 / \delta}\right)\right\} \\
& \Rightarrow \varrho\left(\mathscr{F}_{n t_{(1)}} \oplus \mathscr{F}_{n t_{(2)}}\right)=\varrho \mathscr{F}_{n t_{(1)}} \oplus \varrho \mathscr{F}_{n t_{(2)}} \\
& \varrho_{1} \mathscr{F}_{n t_{(1)}} \oplus \varrho_{2} \mathscr{F}_{n t(1)}=\left\{\min \left(1,\left(\varrho_{1} b_{1}^{\delta}\right)^{1 / \delta}\right), 1-\min \left(1,\left(\varrho_{1}\left(1-I_{1}\right)^{\delta}\right)^{1 / \delta}\right), 1-\min \left(1,\left(\varrho_{1}\left(1-\partial_{1}\right)^{\delta}\right)^{1 / \delta}\right)\right\} \oplus\left\{\min \left(1,\left(\varrho_{2} b_{2}^{\delta}\right)^{1 / \delta}\right), 1\right. \\
& \left.-\min \left(1,\left(\varrho_{2}\left(1-I_{2}\right)^{\delta}\right)^{1 / \delta}\right), 1-\min \left(1,\left(\varrho_{2}\left(1-\partial_{2}\right)^{\delta}\right)^{1 / \delta}\right)\right\} \\
& =\left\{\min \left(1,\left(\left(\varrho_{1}+\varrho_{2}\right) b_{1}^{\delta}\right)^{1 / \delta}\right), 1-\min \left(1,\left(\left(\varrho_{1}+\varrho_{2}\right)\left(1-I_{1}\right)^{\delta}\right)^{1 / \delta}\right), 1-\min \left(1,\left(\left(\varrho_{1}+\varrho_{2}\right)\left(1-\partial_{1}\right)^{\delta}\right)^{1 / \delta}\right)\right\} \\
& =\left(\varrho_{1}+\varrho_{2}\right) \mathscr{F}_{n t(1)} \text {. }
\end{aligned}
$$


Proof of (5) and (6) are similar as above.

\section{Aggregation Operators Based on Yager's Norms}

The section presents some single-valued neutrosophic AgOs using Yager OLs of SVNNs.

\subsection{Yager Weighted Averaging AgOs}

Definition 14. Let $\mathscr{F}_{h}=\left(b_{h}(x), I_{h}(x), \partial_{h}(x)\right) \in \operatorname{SVNN}(U)$ $(h \in \mathbb{N})$. Then, Yager weighted averaging AgOs for $\operatorname{SVNN}(U)$ is described as follows:

$$
\begin{aligned}
\operatorname{SVNYWA}\left(\mathscr{F}_{1}, \mathscr{F}_{2}, \ldots, \mathscr{F}_{n}\right) & =\rho_{1} \mathscr{F}_{1} \oplus \rho_{2} \mathscr{F}_{2} \oplus \cdots \oplus \rho_{n} \mathscr{F}_{n} \\
& =\sum_{h=1}^{n} \rho_{h} \mathscr{F}_{h},
\end{aligned}
$$

where the weights $\left(\rho_{1}, \rho_{2}, \ldots, \rho_{h}\right)$ of $\mathscr{F}_{h}$ have $\rho_{h} \geq 0$ and $\sum_{h=1}^{n} \rho_{h}=1$.

Theorem 2. Let $\quad \mathscr{F}_{h}=\left(b_{h}(x), I_{h}(x), \partial_{h}(x)\right) \in S V N N$ $(U)(h \in \mathbb{N})$ and the weights $\left(\rho_{1}, \rho_{2}, \ldots, \rho_{h}\right)$ of $\mathscr{F}_{h}$ having $\rho_{h} \geq 0$ and $\sum_{h=1}^{n} \rho_{h}=1$ The SVNYWA AgOs are a mapping $\mathscr{G}^{n} \longrightarrow \mathscr{G}$ such that

$$
\begin{aligned}
\operatorname{SVNYWA}\left(\mathscr{F}_{1}, \mathscr{F}_{2}, \ldots, \mathscr{F}_{n}\right)= & \sum_{h=1}^{n} \rho_{h} \mathscr{F}_{h} \\
= & \left(\min \left(1,\left(\sum_{h=1}^{n} \rho_{h} b_{h}^{\delta}\right)^{1 / \delta}\right), 1-\min \left(1,\left(\sum_{h=1}^{n} \rho_{h}\left(1-I_{h}\right)^{\delta}\right)^{1 / \delta}\right), 1\right. \\
& \left.-\min \left(1,\left(\sum_{h=1}^{n} \rho_{h}\left(1-\partial_{h}\right)^{\delta}\right)^{1 / \delta}\right)\right) .
\end{aligned}
$$

Proof. We prove Theorem 2, by applying mathematical induction on $n$, since for each $h$, $\mathscr{F}_{h}=\left(b_{h}(x), I_{h}(x), \partial_{h}(x)\right) \in \operatorname{SVNN}(U)$ which implies that

$$
\operatorname{SVNYWA}\left(\mathscr{F}_{1}, \mathscr{F}_{2}\right)=\rho_{1} \mathscr{F}_{1} \oplus \rho_{2} \mathscr{F}_{2} .
$$
$b_{h}, I_{h}, \partial_{h} \in[0,1]$ and $b_{h}+I_{h}+\partial_{h} \leq 3$.

Step 1: for $n=2$, we obtain

$$
\begin{aligned}
\operatorname{SVNYWA}\left(\mathscr{F}_{1}, \mathscr{F}_{2}\right)= & \rho_{1} \mathscr{F}_{1} \oplus \rho_{2} \mathscr{F}_{2} \\
= & \left\{\min \left(1,\left(\rho_{1} b_{1}^{\delta}\right)^{1 / \delta}\right), 1-\min \left(1,\left(\rho_{1}\left(1-I_{1}\right)^{\delta}\right)^{1 / \delta}\right), 1-\min \left(1,\left(\rho_{1}\left(1-\partial_{1}\right)^{\delta}\right)^{1 / \delta}\right)\right\} \\
& \cdot \oplus\left\{\min \left(1,\left(\rho_{2} b_{2}^{\delta}\right)^{1 / \delta}\right), 1-\min \left(1,\left(\rho_{2}\left(1-I_{2}\right)^{\delta}\right)^{1 / \delta}\right), 1-\min \left(1,\left(\rho_{2}\left(1-\partial_{2}\right)^{\delta}\right)^{1 / \delta}\right)\right\} \\
= & \left(\min \left(1,\left(\sum_{h=1}^{2} \rho_{h} b_{h}^{\delta}\right)^{1 / \delta}\right), 1-\min \left(1,\left(\sum_{h=1}^{2} \rho_{h}\left(1-I_{h}\right)^{\delta}\right)^{1 / \delta}\right), 1-\min \left(1,\left(\sum_{h=1}^{2} \rho_{h}\left(1-\partial_{h}\right)^{\delta}\right)\right) .\right.
\end{aligned}
$$


Step 2: suppose that equation (11) holds for $n=\kappa$, and we have

$$
\begin{aligned}
\operatorname{SVNYWA}\left(\mathscr{F}_{1}, \mathscr{F}_{2}, \ldots, \mathscr{F}_{\kappa}\right) & =\left(\sqrt{\min \left(1,\left(\sum_{h=1}^{\kappa} \rho_{h} b_{h}^{2 \delta}\right)^{1 / \delta}\right)}, \sqrt{1-\min \left(1,\left(\sum_{h=1}^{\kappa} \rho_{h}\left(1-I_{h}^{2}\right)^{\delta}\right)^{1 / \delta}\right)}\right. \\
& \cdot \sqrt{1-\min \left(1,\left(\sum_{h=1}^{\kappa} \rho_{h}\left(1-\partial_{h}^{2}\right)^{\delta}\right)^{1 / \delta}\right)} .
\end{aligned}
$$

Step 3: now, we have to prove that equation (11) holds for $n=\kappa+1$ :

$$
\begin{aligned}
\operatorname{SVNYWA}\left(\mathscr{F}_{1}, \mathscr{F}_{2}, \ldots, \mathscr{F}_{\kappa+1}\right)= & \sum_{h=1}^{\kappa} \rho_{h} \mathscr{F}_{h} \oplus \rho_{\kappa+1} \mathscr{F}_{\kappa+1} \\
= & \left(\min \left(1,\left(\sum_{h=1}^{\kappa} \rho_{h} b_{h}^{\delta}\right)^{1 / \delta}\right), 1-\min \left(1,\left(\sum_{h=1}^{\kappa} \rho_{h}\left(1-I_{h}\right)^{\delta}\right)^{1 / \delta}\right), 1\right. \\
& \left.-\min \left(1,\left(\sum_{h=1}^{\kappa} \rho_{h}\left(1-\partial_{h}\right)^{\delta}\right)^{1 / \delta}\right)\right) \oplus\left(\min \left(1,\left(\rho_{\kappa+1} b_{\kappa+1}^{\delta}\right)^{1 / \delta}\right), 1\right. \\
& \left.-\min \left(1,\left(\rho_{\kappa+1}\left(1-I_{\kappa+1}\right)^{\delta}\right)^{1 / \delta}\right), 1-\min \left(1,\left(\rho_{\kappa+1}\left(1-\partial_{\kappa+1}\right)^{\delta}\right)^{1 / \delta}\right)\right) \\
= & \left(\min \left(1,\left(\sum_{h=1}^{\kappa+1} \rho_{h} b_{h}^{\delta}\right)^{1 / \delta}\right)^{1 / \delta}, 1-\min \left(1,\left(\sum_{h=1}^{\kappa+1} \rho_{h}\left(1-I_{h}\right)^{\delta}\right)^{1 / \delta}, 1\right.\right. \\
& -\min \left(1,\left(\sum_{h=1}^{\kappa+1} \rho_{h}\left(1-\partial_{h}\right)^{\delta}\right)\right),
\end{aligned}
$$

that is, when $n=z+1$, equation (11) also holds.

Hence, equation (11) holds for any $n$. The proof is completed.

Next, we give the some properties of the proposed SVNYWA aggregation operator.
Theorem 3. Let $\mathscr{F}_{h}=\left(b_{h}(x), I_{h}(x), \partial_{h}(x)\right) \in S V N N$ $(U)(h \in \mathbb{N})$ such that $\mathscr{F}_{h}=\mathscr{F}$. Then,

$$
\operatorname{SVNYWA}\left(\mathscr{F}_{1}, \mathscr{F}_{2}, \ldots, \mathscr{F}_{n}\right)=\mathscr{F}
$$


Proof. Since $\mathscr{F}_{h}=\mathscr{F}(h \in \mathbb{N})$. Then, by Theorem 2, we obtain

$$
\begin{aligned}
\operatorname{SVNYWA}\left(\mathscr{F}_{1}, \mathscr{F}_{2}, \ldots, \mathscr{F}_{n}\right)= & \left(\min \left(1,\left(\sum_{h=1}^{n} \rho_{h} b_{h}^{2 \delta}\right)^{1 / \delta}\right), 1-\min \left(1,\left(\sum_{h=1}^{n} \rho_{h}\left(1-I_{h}\right)^{\delta}\right)^{1 / \delta}\right), 1\right. \\
& \left.-\min \left(1,\left(\sum_{h=1}^{n} \rho_{h}\left(1-\partial_{h}\right)^{\delta}\right)^{1 / \delta}\right)\right) \\
= & \left(\min \left(1,\left(\sum_{h=1}^{n} \rho_{h} b^{\delta}\right)^{1 / \delta}\right), 1-\min \left(1,\left(\sum_{h=1}^{n} \rho_{h}(1-I)^{\delta}\right)^{1 / \delta}\right), 1\right. \\
& -\min \left(1,\left(\sum_{h=1}^{n} \rho_{h}(1-\partial)^{\delta}\right)\right) \\
= & \left(\min \left(1,\left(b^{\delta}\right)^{1 / \delta}\right), 1-\min \left(1,\left((1-I)^{\delta}\right)^{1 / \delta}\right), 1-\min \left(1,\left((1-\partial)^{\delta}\right)^{1 / \delta}\right)\right) \\
= & (b(x), I(x), \partial(x)) \\
= & \mathscr{F} .
\end{aligned}
$$

Hence, proved.

Theorem 4. Let $\mathscr{F}_{h}=\left(b_{h}(x), I_{h}(x), \partial_{h}(x)\right), \mathscr{F}_{h}^{-}=\{\min$ $\left.\left(b_{h}(x)\right), \quad \max \left(I_{h}(x)\right), \max \left(\partial_{h}(x)\right)\right\}$ and $\mathscr{F}_{h}^{+}=\left\{\max \left(b_{h}\right.\right.$ $\left.(x)), \quad \min \left(I_{h}(x)\right), \min \left(\partial_{h}(x)\right)\right\} \in \operatorname{SVNN}(U) \quad(h \in \mathbb{N})$. Then,

$$
\mathscr{F}_{h}^{-} \leq \operatorname{SVNYWA}\left(\mathscr{F}_{1}, \mathscr{F}_{2}, \ldots, \mathscr{F}_{n}\right) \leq \mathscr{F}_{h}^{+} .
$$

Proof. Procedure is similar as the above theorem, so here we eliminate.
Theorem 5. Let $\mathscr{F}_{h}=\left(b_{h}(x), I_{h}(x), \partial_{h}(x)\right), \mathscr{F}_{h}^{*}=\left(b_{h}^{*}(x)\right.$, $\left.I_{h}^{*}(x), \partial_{h}^{*}(x)\right) \in \operatorname{SVNN}(U)(h \in \mathbb{N})$. If $b_{h} \leq b_{h}^{*}, I_{h} \leq I_{h}^{*}$, and $\partial_{h} \leq \partial_{h}^{*}$, then

$$
\operatorname{SVNYWA}\left(\mathscr{F}_{1}, \mathscr{F}_{2}, \ldots, \mathscr{F}_{n}\right) \leq \operatorname{SVNYWA}\left(\mathscr{F}_{1}^{*}, \mathscr{F}_{2}^{*}, \ldots, \mathscr{F}_{n}^{*}\right) .
$$

Proof. Procedure is similar as above theorem, so here we eliminate.

Definition 15. Let $\mathscr{F}_{h}=\left(b_{h}(x), I_{h}(x), \partial_{h}(x)\right) \in \operatorname{SVNN}(U)$ $(h \in \mathbb{N})$. Then, Yager ordered weighted averaging AgOs for $\operatorname{SVNN}(U)$ is described as follows:

$$
\begin{aligned}
\operatorname{SVNYOWA}\left(\mathscr{F}_{1}, \mathscr{F}_{2}, \ldots, \mathscr{F}_{n}\right) & =\rho_{1} \mathscr{F}_{x(1)} \oplus \rho_{2} \mathscr{F}_{x(2)} \oplus \cdots \oplus \rho_{n} \mathscr{F}_{x(n)} \\
& =\sum_{h=1}^{n} \rho_{h} \mathscr{F}_{x(h)},
\end{aligned}
$$

where $x(h)$ represented the ordered and $(x(1), x(2), x(3), \ldots, x(n))$ is a permutation of $(1,2,3, \ldots, n)$, subject to $\varepsilon_{x(h-1)} \geq \varepsilon_{x(h)}$ for all $h$. Also, the weights $\left(\rho_{1}, \rho_{2}, \ldots, \rho_{h}\right)$ of $\mathscr{F}_{h}$ having $\rho_{h} \geq 0$ and $\sum_{h=1}^{n} \rho_{h}=1$.
Theorem 6. Let $\mathscr{F}_{h}=\left(b_{h}(x), I_{h}(x), \partial_{h}(x)\right) \in \quad$ SVNN $(U)(h \in \mathbb{N})$ and the weights $\left(\rho_{1}, \rho_{2}, \ldots, \rho_{h}\right)$ of $\mathscr{F}_{h}$ having $\rho_{h} \geq 0$ and $\sum_{h=1}^{n} \rho_{h}=1$ The SVNYOWA AgOs is a mapping $\mathscr{G}^{n} \longrightarrow \mathscr{G}$ such that 


$$
\begin{aligned}
\operatorname{SVNYOWA}\left(\mathscr{F}_{1}, \mathscr{F}_{2}, \ldots, \mathscr{F}_{n}\right)= & \sum_{h=1}^{n} \rho_{h} \mathscr{F}_{x(h)} \\
= & \left(\min \left(1,\left(\sum_{h=1}^{n} \rho_{h} b_{x(h)}^{\delta}\right)^{1 / \delta}\right), 1-\min \left(1,\left(\sum_{h=1}^{n} \rho_{h}\left(1-I_{x(h)}\right)^{\delta}\right), 1\right.\right. \\
& \left.-\min \left(1,\left(\sum_{h=1}^{n} \rho_{h}\left(1-\partial_{x(h)}\right)^{\delta}\right)^{1 / \delta}\right)\right) .
\end{aligned}
$$

Proof. It follows from Theorem 2 similarly.

Theorem 7. Let $\mathscr{F}_{h}=\left(b_{h}(x), I_{h}(x), \partial_{h}(x)\right) \in \operatorname{SVNN}(U)$ $(h \in \mathbb{N})$ such that $\mathscr{F}_{h}=\mathscr{F}$. Then,

$$
\operatorname{SVNYOWA}\left(\mathscr{F}_{1}, \mathscr{F}_{2}, \ldots, \mathscr{F}_{n}\right)=\mathscr{F} \text {. }
$$

Theorem 8. Let $\mathscr{F}_{h}=\left(b_{h}(x), I_{h}(x), \partial_{h}(x)\right), \mathscr{F}_{h}^{-}=\{\min$ $\left.\left(b_{h}(x)\right), \max \left(I_{h}(x)\right), \max \left(\partial_{h}(x)\right)\right\}, \quad$ and $\mathscr{F}_{h}^{+}=\{\max$ $\left.\left(b_{h}(x)\right), \min \left(I_{h}(x)\right), \min \left(\partial_{h}(x)\right)\right\} \in \operatorname{SVNN}(U)(h \in \mathbb{N})$. Then,

$$
\mathscr{F}_{h}^{-} \leq \operatorname{SVNYOWA}\left(\mathscr{F}_{1}, \mathscr{F}_{2}, \ldots, \mathscr{F}_{n}\right) \leq \mathscr{F}_{h}^{+} .
$$

Theorem 9. Let $\mathscr{F}_{h}=\left(b_{h}(x), I_{h}(x), \partial \quad{ }_{h}(x)\right)$ and $\mathscr{F}_{h}^{*}=$ $\left(b_{h}^{*}(x), I_{h}^{*}(x), \partial_{h}^{*}(x)\right) \in \operatorname{SVNN}(U)(h \in \mathbb{N})$.

$b_{h} \leq b_{h}^{*}, I_{h} \leq I_{h}^{*}$, and $\partial_{h} \leq \partial_{h}^{*}$, then

$\operatorname{SVNYOWA}\left(\mathscr{F}_{1}, \mathscr{F}_{2}, \ldots, \mathscr{F}_{n}\right) \leq \operatorname{SVNYOWA}\left(\mathscr{F}_{1}^{*}, \mathscr{F}_{2}^{*}, \ldots, \mathscr{F}_{n}^{*}\right)$.

Proof of these theorems is similarly followed by Theorems 3-5.

Definition 16. Let $\mathscr{F}_{h}=\left(b_{h}(x), I_{h}(x), \partial_{h}(x)\right) \in \operatorname{SVNN}(U)$ $(h \in \mathbb{N})$. Then, Yager hybrid weighted averaging AgOs for $\operatorname{SVNN}(U)$ is described as follows:

$$
\begin{aligned}
\operatorname{SVNYHWA}\left(\mathscr{F}_{1}, \mathscr{F}_{2}, \ldots, \mathscr{F}_{n}\right) & =\sigma_{h} \mathscr{F}_{x(1)}^{\prime} \oplus \sigma_{h} \mathscr{F}_{x(2)}^{\prime} \oplus \ldots \oplus \sigma_{h} \mathscr{F}_{x(n)}^{\prime} \\
& =\sum_{h=1}^{n} \sigma_{h} \mathscr{F}_{x(h)}^{\prime},
\end{aligned}
$$

where weights $\left(\rho_{1}, \rho_{2}, \ldots, \rho_{h}\right)$ of $\mathscr{F}_{h}$ having $\rho_{h} \geq 0$ and $\sum_{h=1}^{n} \rho_{h}=1$ and $g$ th biggest weighted value is $\mathscr{F}_{x(h)}^{\prime}\left(\mathscr{F}_{x(h)}^{\prime}=n \rho_{h} \mathscr{F}_{x(h)} \mid h=1,2, \ldots, n\right)$, consequently by total order $(x(1), x(2), x(3), \ldots, x(n))$. Also, associated weights $\left(\sigma_{1}, \sigma_{2}, \ldots, \sigma_{h}\right)$ of $\mathscr{F}_{h}$ having $\sigma_{h} \geq 0$ and $\sum_{h=1}^{n} \sigma_{h}=1$.
Theorem 10. Let $\mathscr{F}_{h}=\left(b_{h}(x), I_{h}(x), \partial_{h}(x)\right) \in \operatorname{SVNN}(U)$ $(h \in \mathbb{N})$ and the weights $\left(\rho_{1}, \rho_{2}, \ldots, \rho_{h}\right)$ of $\mathscr{F}_{h}$ having $\rho_{h} \geq 0$ and $\sum_{h=1}^{n} \rho_{h}=1$ The SVNYHWA AgOs are a mapping $\mathscr{G}^{n} \longrightarrow \mathscr{G}$ with associated weights $\left(\sigma_{1}, \sigma_{2}, \ldots, \sigma_{h}\right)$ of $\mathscr{F}_{h}$ having $\sigma_{h} \geq 0$ and $\sum_{h=1}^{n} \sigma_{h}=1$, and we have

$$
\begin{aligned}
\operatorname{SFYHWA}\left(\mathscr{F}_{1}, \mathscr{F}_{2}, \ldots, \mathscr{F}_{n}\right)= & \sum_{h=1}^{n} \sigma_{h} \mathscr{F}_{x(h)}^{\prime} \\
= & \left(\min \left(1,\left(\sum_{h=1}^{n} \rho_{h} b_{x(h)}^{\prime \delta}\right)^{1 / \delta}\right), 1-\min \left(1,\left(\sum_{h=1}^{n} \rho_{h}\left(1-I_{x(h)}^{\prime}\right)^{\delta}\right), 1\right.\right. \\
& \left.-\min \left(1,\left(\sum_{h=1}^{n} \rho_{h}\left(1-\partial_{x(h)}^{\prime}\right)^{\delta}\right)^{1 / \delta}\right)\right) .
\end{aligned}
$$

Proof. It follows from Theorem 2 similarly.

Theorem 11. Let $\mathscr{F}_{h}=\left(b_{h}(x), I_{h}(x), \partial_{h}(x)\right) \in \operatorname{SVNN}(U)$ $(h \in \mathbb{N})$ such that $\mathscr{F}_{h}=\mathscr{F}$. Then,

$$
\operatorname{SVNYHWA}\left(\mathscr{F}_{1}, \mathscr{F}_{2}, \ldots, \mathscr{F}_{n}\right)=\mathscr{F} \text {. }
$$

Theorem 12. Let $\mathscr{F}_{h}=\left(b_{h}(x), I_{h}(x), \partial_{h}(x)\right), \mathscr{F}_{h}^{-}=\{\min$ $\left.\left(b_{h}(x)\right), \max \left(I_{h}(x)\right), \max \left(\partial_{h}(x)\right)\right\}$ $\mathscr{F}_{h}^{+}=\left\{\max \left(b_{h}(x)\right), \min \left(I_{h}(x)\right), \min \quad\left(\partial_{h}(x)\right)\right\} \in S V N N$ $(U)(h \in \mathbb{N})$. Then,

$$
\mathscr{F}_{h}^{-} \leq \operatorname{SVNYHWA}\left(\mathscr{F}_{1}, \mathscr{F}_{2}, \ldots, \mathscr{F}_{n}\right) \leq \mathscr{F}_{h}^{+} .
$$


Theorem 13. Let $\mathscr{F}_{h}=\left(b_{h}(x), I_{h}(x), \partial_{h}(x)\right), \mathscr{F}_{h}^{*}=\left(b_{h}^{*}(x)\right.$, $\left.I_{h}^{*}(x), \partial_{h}^{*}(x)\right) \in \operatorname{SVNN}(U)(h \in \mathbb{N})$. If $b_{h} \leq b_{h}^{*}, I_{h} \leq I_{h}^{*}$ and $\partial_{h} \leq \partial_{h}^{*}$, then

$$
\operatorname{SVNYHWA}\left(\mathscr{F}_{1}, \mathscr{F}_{2}, \ldots, \mathscr{F}_{n}\right) \leq \operatorname{SVNYHWA}\left(\mathscr{F}_{1}^{*}, \mathscr{F}_{2}^{*}, \ldots, \mathscr{F}_{n}^{*}\right) .
$$

Proof of these theorems is similarly followed by Theorems 3-5.

\subsection{Yager Weighted Geometric AgOs}

Definition 17. Let $\mathscr{F}_{h}=\left(b_{h}(x), I_{h}(x), \partial_{h}(x)\right) \in \operatorname{SVNN}(U)$ $(h \in \mathbb{N})$. Then, Yager weighted geometric AgOs for $\operatorname{SVNN}(U)$ is described as follows:

$$
\begin{aligned}
\operatorname{SVNYWG}\left(\mathscr{F}_{1}, \mathscr{F}_{2}, \ldots, \mathscr{F}_{n}\right) & =\mathscr{F}_{1}^{\rho_{1}} \otimes \mathscr{F}_{2}^{\rho_{2}} \otimes \cdots \otimes \mathscr{F}_{n}^{\rho_{n}} \\
& =\prod_{h=1}^{n}\left(\mathscr{F}_{h}\right)^{\rho_{h}},
\end{aligned}
$$

where the weights $\left(\rho_{1}, \rho_{2}, \ldots, \rho_{h}\right)$ of $\mathscr{F}_{h}$ having $\rho_{h} \geq 0$ and $\sum_{h=1}^{n} \rho_{h}=1$.

Theorem 14. Let $\mathscr{F}_{h}=\left(b_{h}(x), I_{h}(x), \partial_{h}(x)\right) \in \operatorname{SVNN}(U)$ $(h \in \mathbb{N})$ and the weights $\left(\rho_{1}, \rho_{2}, \ldots, \rho_{h}\right)$ of $\mathscr{F}_{h}$ having $\rho_{h} \geq 0$ and $\sum_{h=1}^{n} \rho_{h}=1$ The SVNYWG AgOs is a mapping $\mathscr{G}^{n} \longrightarrow \mathscr{G}$ such that

$$
\begin{aligned}
\operatorname{SVNYWG}\left(\mathscr{F}_{1}, \mathscr{F}_{2}, \ldots, \mathscr{F}_{n}\right) & =\prod_{h=1}^{n}\left(\mathscr{F}_{h}\right)^{\rho_{h}} \\
& =\left(1-\min \left(1,\left(\sum_{h=1}^{n} \rho_{h}\left(1-b_{h}\right)^{\delta}\right)^{1 / \delta}\right), \min \left(1,\left(\sum_{h=1}^{n} \rho_{h} I_{h}^{\delta}\right)^{1 / \delta}\right), \min \left(1,\left(\sum_{h=1}^{n} \rho_{h} \partial_{h}^{\delta}\right)^{1 / \delta}\right)\right) .
\end{aligned}
$$

$$
\operatorname{SVNYWG}\left(\mathscr{F}_{1}, \mathscr{F}_{2}\right)=\mathscr{F}_{1}^{\rho_{1}} \otimes \mathscr{F}_{2}^{\rho_{2}} .
$$

Proof. We prove Theorem 14, by applying mathematical induction on $n$. Since for each $h$, $\mathscr{F}_{h}=\left(b_{h}(x), I_{h}(x), \partial_{h}(x)\right) \in \operatorname{SVNN}(U)$ which implies that Since by Definition 13, we have $b_{h}, I_{h}, \partial_{h} \in[0,1]$ and $b_{h}+I_{h}+\partial_{h} \leq 3$.

Step 1: for $n=2$, we obtain

$$
\begin{aligned}
\operatorname{SVNYWG}\left(\mathscr{F}_{1}, \mathscr{F}_{2}\right)= & \mathscr{F}_{1}^{\rho_{1}} \otimes \mathscr{F}_{2}^{\rho_{2}} \\
= & \left\{1-\min \left(1,\left(\rho_{1}\left(1-b_{1}\right)^{\delta}\right)^{1 / \delta}\right), \min \left(1,\left(\rho_{1} I_{1}^{\delta}\right)^{1 / \delta}\right), \min \left(1,\left(\rho_{1} \partial_{1}^{\delta}\right)^{1 / \delta}\right)\right\} \\
& \oplus\left\{1-\min \left(1,\left(\rho_{2}\left(1-b_{2}\right)^{\delta}\right)^{1 / \delta}\right), \min \left(1,\left(\rho_{2} I_{2}^{\delta}\right)^{1 / \delta}\right), \min \left(1,\left(\rho_{2} \partial_{2}^{\delta}\right)^{1 / \delta}\right)\right\} \\
& =\left(1-\min \left(1,\left(\sum_{h=1}^{2} \rho_{h}\left(1-b_{h}\right)^{\delta}\right)^{1 / \delta}\right), \min \left(1,\left(\sum_{h=1}^{2} \rho_{h} I_{h}^{\delta}\right)^{1 / \delta}\right), \min \left(1,\left(\sum_{h=1}^{2} \rho_{h} \partial_{h}^{\delta}\right)^{1 / \delta}\right)\right) .
\end{aligned}
$$

Step 2: suppose that equation (31) holds for $n=\kappa$, and we have

$\operatorname{SVNYWG}\left(\mathscr{F}_{1}, \mathscr{F}_{2}, \ldots, \mathscr{F}_{\kappa}\right)=\left(1-\min \left(1,\left(\sum_{h=1}^{\kappa} \rho_{h}\left(1-b_{h}\right)^{\delta}\right)^{1 / \delta}\right), \min \left(1,\left(\sum_{h=1}^{\kappa} \rho_{h} I_{h}^{\delta}\right)^{1 / \delta}\right), \min \left(1,\left(\sum_{h=1}^{\kappa} \rho_{h} \partial_{h}^{\delta}\right)^{1 / \delta}\right)\right)$ 
Step 3: now, we have to prove that equation (31) holds for $n=\kappa+1$ :

$$
\begin{aligned}
\operatorname{SVNYWG}\left(\mathscr{F}_{1}, \mathscr{F}_{2}, \ldots, \mathscr{F}_{\kappa+1}\right)= & \prod_{h=1}^{\kappa}\left(\mathscr{F}_{h}\right)^{\rho_{h}} \otimes\left(\mathscr{F}_{\kappa+1}\right)^{\rho_{\kappa+1}} \\
= & \left(1-\min \left(1,\left(\sum_{h=1}^{\kappa} \rho_{h}\left(1-b_{h}\right)^{\delta}\right)^{1 / \delta}\right), \min \left(1,\left(\sum_{h=1}^{\kappa} \rho_{h} I_{h}^{\delta}\right)^{1 / \delta}\right), \min \left(1,\left(\sum_{h=1}^{\kappa} \rho_{h} \partial_{h}^{\delta}\right)\right)\right. \\
& \oplus\left\{1-\min \left(1,\left(\rho_{\kappa+1}\left(1-b_{\kappa+1}\right)^{\delta}\right)^{1 / \delta}\right), \min \left(1,\left(\rho_{\kappa+1} I_{\kappa+1}^{\delta}\right)^{1 / \delta}\right), \min \left(1,\left(\rho_{\kappa+1} \partial_{\kappa+1}^{\delta}\right)^{1 / \delta}\right)\right\} \\
= & \left(1-\min \left(1,\left(\sum_{h=1}^{\kappa+1} \rho_{h}\left(1-b_{h}\right)^{\delta}\right)^{1 / \delta}\right), \min \left(1,\left(\sum_{h=1}^{\kappa+1} \rho_{h} I_{h}^{\delta}\right)^{1 / \delta}\right), \min \left(1,\left(\sum_{h=1}^{\kappa+1} \rho_{h} \partial_{h}^{\delta}\right)^{1 / \delta}\right),\right.
\end{aligned}
$$

$$
\operatorname{SVNYWG}\left(\mathscr{F}_{1}, \mathscr{F}_{2}, \ldots, \mathscr{F}_{n}\right)=\mathscr{F}
$$

that is, when $n=z+1$, equation (31) also holds.

Hence, equation (31) holds for any $n$. The proof is completed.

Theorem 15. Let $\mathscr{F}_{h}=\left(b_{h}(x), I_{h}(x), \partial_{h}(x)\right) \in \operatorname{SVNN}(U)$ $(h \in \mathbb{N})$ such that $\mathscr{F}_{h}=\mathscr{F}$. Then,
Proof. Since $\mathscr{F}_{h}=\mathscr{F}(h \in \mathbb{N})$. Then, by Theorem 14, we obtain

$$
\begin{aligned}
\operatorname{SVNYWG}\left(\mathscr{F}_{1}, \mathscr{F}_{2}, \ldots, \mathscr{F}_{n}\right) & \left.=\left(1-\min \left(1,\left(\sum_{h=1}^{n} \rho_{h}\left(1-b_{h}\right)^{\delta}\right)^{1 / \delta}\right), \min \left(1,\left(\sum_{h=1}^{n} \rho_{h} I_{h}^{\delta}\right)^{1 / \delta}\right), \min \left(1,\left(\sum_{h=1}^{n} \rho_{h} \partial_{h}^{\delta}\right)^{1 / \delta}\right)\right), \min \left(1,\left(\sum_{h=1}^{n} \rho_{h} I^{\delta}\right)^{1 / \delta}\right), \min \left(1,\left(\sum_{h=1}^{n} \rho_{h} \partial^{\delta}\right)^{1 / \delta}\right)\right) \\
& =\left(1-\min \left(1,\left(\sum_{h=1}^{n} \rho_{h}(1-b)^{\delta}\right)^{1 / \delta}\right) \min (1,(\partial))\right) \\
& =(1-\min (1,(1-b)), \min (1,(I)),(x)) \\
& =(b(x), I(x), \partial(x)) \\
& =\mathscr{F} .
\end{aligned}
$$

Hence, proved.

$$
\mathscr{F}_{h}^{-} \leq \operatorname{SVNYWG}\left(\mathscr{F}_{1}, \mathscr{F}_{2}, \ldots, \mathscr{F}_{n}\right) \leq \mathscr{F}_{h}^{+} .
$$

Theorem 16. Let $\mathscr{F}_{h}=\left(b_{h}(x), I_{h}(x), \partial_{h}(x)\right), \mathscr{F}_{h}^{-}=\{\min$ $\left.\left(b_{h}(x)\right), \max \left(I_{h}(x)\right), \max \left(\partial_{h}(x)\right)\right\} \quad$ and $\mathscr{F}_{h}^{+}=\left\{\max \left(b_{h}\right.\right.$ $\left.(x)), \min \left(I_{h}(x)\right), \min \left(\partial_{h}(x)\right)\right\} \in S V N N(U)(h \in \mathbb{N})$. Then,
Theorem 17. Let $\mathscr{F}_{h}=\left(b_{h}(x), I_{h}(x), \partial_{h}(x)\right), \mathscr{F}_{h}^{*}=\left(b_{h}^{*}(x)\right.$, $\left.I_{h}^{*}(x), \partial_{h}^{*}(x)\right) \in \operatorname{SVNN}(U)(h \in \mathbb{N})$. If $b_{h} \leq b_{h}^{*}, I_{h} \leq I_{h}^{*}$, and $\partial_{h} \leq \partial_{h}^{*}$, then 
$\operatorname{SVNYWG}\left(\mathscr{F}_{1}, \mathscr{F}_{2}, \ldots, \mathscr{F}_{n}\right) \leq \operatorname{SVNYWG}\left(\mathscr{F}_{1}^{*}, \mathscr{F}_{2}^{*}, \ldots, \mathscr{F}_{n}^{*}\right)$
Definition 18. Let $\mathscr{F}_{h}=\left(b_{h}(x), I_{h}(x), \partial_{h}(x)\right) \in \operatorname{SVNN}(U)$ $(h \in \mathbb{N})$. Then, Yager ordered weighted geometric AgOs for $\operatorname{SVNN}(U)$ is described as follows:

$$
\begin{aligned}
\operatorname{SVNYOWG}\left(\mathscr{F}_{1}, \mathscr{F}_{2}, \ldots, \mathscr{F}_{n}\right) & =\left(\mathscr{F}_{x(1)}\right)^{\rho_{1}} \otimes\left(\mathscr{F}_{x(2)}\right)^{\rho_{2}} \otimes \cdots \otimes\left(\mathscr{F}_{x(n)}\right)^{\rho_{n}} \\
& =\prod_{h=1}^{n}\left(\mathscr{F}_{x(h)}\right)^{\rho_{h}},
\end{aligned}
$$

where $x(h)$ represented the ordered and $(x(1), x(2), x(3), \ldots, x(n))$ is a permutation of $(1,2,3, \ldots, n)$, subject to $\varepsilon_{x(h-1)} \geq \varepsilon_{x(h)}$ for all $h$. Also, the weights $\left(\rho_{1}, \rho_{2}, \ldots, \rho_{h}\right)$ of $\mathscr{F}_{h}$ have $\rho_{h} \geq 0$ and $\sum_{h=1}^{n} \rho_{h}=1$.
Theorem 18. Let $\mathscr{F}_{h}=\left(b_{h}(x), I_{h}(x), \partial_{h}(x)\right) \in S V N N$ $(U)(h \in \mathbb{N})$ and the weights $\left(\rho_{1}, \rho_{2}, \ldots, \rho_{h}\right)$ of $\mathscr{F}_{h}$ have $\rho_{h} \geq 0$ and $\sum_{h=1}^{n} \rho_{h}=1$. The SVNYOWG AgOs is a mapping $\mathscr{G}^{n} \longrightarrow \mathscr{G}$ such that

$$
\begin{aligned}
\operatorname{SVNYOWG}\left(\mathscr{F}_{1}, \mathscr{F}_{2}, \ldots, \mathscr{F}_{n}\right)= & \prod_{h=1}^{n}\left(\mathscr{F}_{x(h)}\right)^{\rho_{h}} \\
= & \left(1-\min \left(1,\left(\sum_{h=1}^{n} \rho_{h}\left(1-b_{x(h)}\right)^{\delta}\right)^{1 / \delta}\right), \min \left(1,\left(\sum_{h=1}^{n} \rho_{h} I_{x(h)}^{\delta}\right)^{1 / \delta}\right),\right. \\
& \left.\cdot \min \left(1,\left(\sum_{h=1}^{n} \rho_{h} \partial_{x(h)}^{\delta}\right)^{1 / \delta}\right)\right) .
\end{aligned}
$$

Proof. It follows from Theorem 14 similarly.

Theorem 19. Let $\mathscr{F}_{h}=\left(b_{h}(x), I_{h}(x), \partial_{h}(x)\right) \in S V N N(U)$ $(h \in \mathbb{N})$ such that $\mathscr{F}_{h}=\mathscr{F}$. Then,

$$
\operatorname{SVNYOWG}\left(\mathscr{F}_{1}, \mathscr{F}_{2}, \ldots, \mathscr{F}_{n}\right)=\mathscr{F} \text {. }
$$

Theorem 20. Let $\mathscr{F}_{h}=\left(b_{h}(x), I_{h}(x), \partial_{h}(x)\right), \mathscr{F}_{h}^{-}=\{\min$ $\left.\left(b_{h}(x)\right), \max \left(I_{h}(x)\right), \max \left(\partial_{h}(x)\right)\right\}$ and $\mathscr{F}_{h}^{+}=\left\{\max \left(b_{h}\right.\right.$ $\left.(x)), \quad \min \left(I_{h}(x)\right), \min \left(\partial_{h}(x)\right)\right\} \in S V N N(U) \quad(h \in \mathbb{N})$. Then,

$$
\mathscr{F}_{h}^{-} \leq \operatorname{SVNYOWG}\left(\mathscr{F}_{1}, \mathscr{F}_{2}, \ldots, \mathscr{F}_{n}\right) \leq \mathscr{F}_{h}^{+} .
$$

Theorem 21. Let $\quad \mathscr{F}_{h}=\left(b_{h}(x), I_{h}(x), \partial \quad{ }_{h}(x)\right), \mathscr{F}_{h}^{*}=$ $\left(b_{h}^{*}(x), I_{h}^{*}(x), \partial_{h}^{*}(x)\right) \in \operatorname{SVNN}(U)(h \in \mathbb{N})$.

$b_{h} \leq b_{h}^{*}, I_{h} \leq I_{h}^{*}$, and $\partial_{h} \leq \partial_{h}^{*}$, then $\operatorname{SVNYOWG}\left(\mathscr{F}_{1}, \mathscr{F}_{2}, \ldots, \mathscr{F}_{n}\right) \leq \operatorname{SVNYOWG}\left(\mathscr{F}_{1}^{*}, \mathscr{F}_{2}^{*}, \ldots, \mathscr{F}_{n}^{*}\right)$.

Proof of these theorems is similarly followed by Theorems $15-17$.

Definition 19. Let $\mathscr{F}_{h}=\left(b_{h}(x), I_{h}(x), \partial_{h}(x)\right) \in \operatorname{SVNN}(U)$ $(h \in \mathbb{N})$. Then, Yager hybrid weighted geometric AgOs for $\operatorname{SVNN}(U)$ is described as follows:

$$
\begin{aligned}
\operatorname{SVNYHWG}\left(\mathscr{F}_{1}, \mathscr{F}_{2}, \ldots, \mathscr{F}_{n}\right) & =\left(\mathscr{F}_{x(1)}^{\prime}\right)^{\sigma_{h}} \otimes\left(\mathscr{F}_{x(2)}^{\prime}\right)^{\sigma_{h}} \otimes \cdots \otimes\left(\mathscr{F}_{x(n)}^{\prime}\right)^{\sigma_{h}} \\
& =\prod_{h=1}^{n}\left(\mathscr{F}_{x(h)}\right)^{\sigma_{h}},
\end{aligned}
$$

where weights $\left(\rho_{1}, \rho_{2}, \ldots, \rho_{h}\right)$ of $\mathscr{F}_{h}$ having $\rho_{h} \geq 0$ and $\sum_{h=1}^{n} \rho_{h}=1$ and $g$ th biggest weighted value is $\mathscr{F}_{x(h)}^{\prime}\left(\mathscr{F}_{x(h)}^{\prime}=n \rho_{h} \mathscr{F}_{x(h)} \mid h=1,2, \ldots, n\right)$, consequently by total order $(x(1), x(2), x(3), \ldots, x(n))$. Also, associated weights $\left(\sigma_{1}, \sigma_{2}, \ldots, \sigma_{h}\right)$ of $\mathscr{F}_{h}$ have $\sigma_{h} \geq 0$ and $\sum_{h=1}^{n} \sigma_{h}=1$.
Theorem 22. Let $\mathscr{F}_{h}=\left(b_{h}(x), I_{h}(x), \partial_{h}(x)\right) \in \operatorname{SVNN}(U)$ $(h \in \mathbb{N})$, and the weights $\left(\rho_{1}, \rho_{2}, \ldots, \rho_{h}\right)$ of $\mathscr{F}_{h}$ have $\rho_{h} \geq 0$ and $\sum_{h=1}^{n} \rho_{h}=1$ The SVNYHWG AgOs is a mapping $\mathscr{G}^{n} \longrightarrow \mathscr{G}$ with associated weights $\left(\sigma_{1}, \sigma_{2}, \ldots, \sigma_{h}\right)$ of $\mathscr{F}_{h}$ having $\sigma_{h} \geq 0$ and $\sum_{h=1}^{n} \sigma_{h}=1$, and we have 


$$
\begin{aligned}
\operatorname{SVNYHWG}\left(\mathscr{F}_{1}, \mathscr{F}_{2}, \ldots, \mathscr{F}_{n}\right)= & \prod_{h=1}^{n}\left(\mathscr{F}_{x(h)}^{\prime}\right)^{\sigma_{h}} \\
= & \left(1-\min \left(1,\left(\sum_{h=1}^{n} \rho_{h}\left(1-b_{x(h)}^{\prime}\right)^{\delta}\right)^{1 / \delta}\right),\right. \\
& \left.\cdot \min \left(1,\left(\sum_{h=1}^{n} \rho_{h} I_{x(h)}^{\prime \delta}\right)^{1 / \delta}\right), \min \left(1,\left(\sum_{h=1}^{n} \rho_{h} \partial_{x(h)}^{\prime \delta}\right)^{1 / \delta}\right)\right) .
\end{aligned}
$$

Proof. It follows from Theorem 14 similarly.

Theorem 23. Let $\mathscr{F}_{h}=\left(b_{h}(x), I_{h}(x), \partial_{h}(x)\right) \in \operatorname{SVNN}(U)$ $(h \in \mathbb{N})$ such that $\mathscr{F}_{h}=\mathscr{F}_{\text {. Then, }}$

$$
\operatorname{SVNYHWG}\left(\mathscr{F}_{1}, \mathscr{F}_{2}, \ldots, \mathscr{F}_{n}\right)=\mathscr{F} \text {. }
$$

Theorem 24. Let $\mathscr{F}=\left(b_{h}(x), I_{h}(x), \partial_{h}(x)\right), \mathscr{F}_{h}^{-}=\{\min$ $\left.\left(b_{h}(x)\right), \max \left(I_{h}(x)\right), \max \left(\partial_{h}(x)\right)\right\}$, and $\mathscr{F}_{h}^{+}=\left\{\max \left(b_{h}(x)\right), \min \left(I_{h}(x)\right), \min \left(\partial_{h}(x)\right)\right\} \in \operatorname{SVNN}(U)$ $(h \in \mathbb{N})$. Then,

$$
\mathscr{F}_{h}^{-} \leq \operatorname{SVNYHWG}\left(\mathscr{F}_{1}, \mathscr{F}_{2}, \ldots, \mathscr{F}_{n}\right) \leq \mathscr{F}_{h}^{+} .
$$

Theorem 25. Let $\mathscr{F}_{h}=\left(b_{h}(x), I_{h}(x), \partial_{h}(x)\right)$ and $\mathscr{F}_{h}^{*}=$ $\left(b_{h}^{*}(x), I_{h}^{*}(x), \partial_{h}^{*}(x)\right) \in \operatorname{SVNN}(U)(h \in \mathbb{N})$. $b_{h} \leq b_{h}^{*}, I_{h} \leq I_{h}^{*}$, and $\partial_{h} \leq \partial_{h}^{*}$, then

$\operatorname{SVNYHWG}\left(\mathscr{F}_{1}, \mathscr{F}_{2}, \ldots, \mathscr{F}_{n}\right) \leq \operatorname{SVNYHWG}\left(\mathscr{F}_{1}^{*}, \mathscr{F}_{2}^{*}, \ldots, \mathscr{F}_{n}^{*}\right)$.
Proof of these theorems is similarly followed by Theorems 15-17.

\section{Algorithm for Decision-Making Problems (DMPs)}

In this section, we propose a framework for solving multiattribute group DMPs under single-valued NS information. Consider a MAGDM with a set of $m$ alternatives $\left\{\beth_{1}, \beth_{2}, \ldots, \beth_{h}\right\}$, and let $\left\{I_{1}, I_{2}, \ldots, I_{h}\right\}$ be a set of attributes with weight vector $\rho=\left(\rho_{1}, \rho_{2}, \ldots, \rho_{h}\right)$, where $\rho_{t} \in[0,1]$ and $\sum_{t=1}^{h} \rho_{t}=1$. To assess the performance of $k$ th alternative $\beth_{k}$ under the $t$ th attribute $I_{t}$, let $\left\{\stackrel{\circ}{D}_{1}, \stackrel{\circ}{D}_{2}, \ldots, \stackrel{\circ}{D}_{\widehat{\xi}}\right\}$ be a set of decision makers and $\widehat{w}=\left(\widehat{w}_{1}, \widehat{w}_{2}, \ldots, \widehat{w}_{\widehat{g}}\right)$ be the weighted vector of decision makers with $\widehat{w}_{s} \in[0,1]$ and $\sum_{s=1}^{\widehat{\mathscr{F}}} \widehat{w}_{s}=1$. The single-valued NS decision matrix can be written as follows:

$$
\left[\begin{array}{cccc}
\left(b_{11}(x), I_{11}(x), \partial_{11}(x)\right) & \left(b_{12}(x), I_{12}(x), \partial_{12}(x)\right) & \cdots & \left(b_{1 h}(x), I_{1 h}(x), \partial_{1 h}(x)\right) \\
\left(b_{21}(x), I_{21}(x), \partial_{21}(x)\right) & \left(b_{22}(x), I_{22}(x), \partial_{22}(x)\right) & \cdots & \left(b_{2 h}(x), I_{2 h}(x), \partial_{2 h}(x)\right) \\
\left(b_{31}(x), I_{31}(x), \partial_{31}(x)\right) & \left(b_{32}(x), I_{32}(x), \partial_{32}(x)\right) & \cdots & \left(b_{3 h}(x), I_{3 h}(x), \partial_{3 h}(x)\right) \\
\vdots & \vdots & \vdots & \vdots \\
\left(b_{h 1}(x), I_{h 1}(x), \partial_{h 1}(x)\right) & \left(b_{h 2}(x), I_{h 2}(x), \partial_{h 2}(x)\right) & \cdots & \left(b_{h h}(x), I_{h h}(x), \partial_{h h}(x)\right)
\end{array}\right],
$$


where $b(x) \in[0,1]$ truth, $I(x) \in[0,1]$ indeterminacy, and $\partial(x) \in[0,1]$ falsity membership grades, respectively. In addition, $0 \leq b(x)+I(x)+\partial(x) \leq 3, \forall x \in U$. Key steps of the developed multiattribute group decision-making (MAGDM) problem are described as follows:
Step 1: construct the single-valued NS decision matrix based on the expert evaluations:

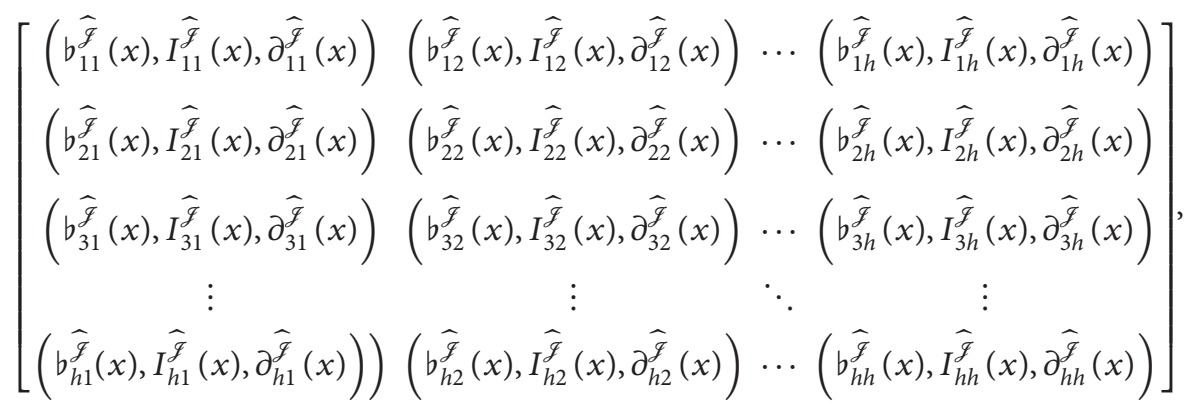

where $\hat{\mathscr{J}}$ represents the number of expert.

Step 2: aggregate the individual decision matrices based on the aggregation operators to construct the aggregated matrix. Hence, the aggregated decision matrix is constructed as follows:

$$
\left[\begin{array}{cccc}
\left(b_{11}(x), I_{11}(x), \partial_{11}(x)\right) & \left(b_{12}(x), I_{12}(x), \partial_{12}(x)\right) & \cdots & \left(b_{1 h}(x), I_{1 h}(x), \partial_{1 h}(x)\right) \\
\left(b_{21}(x), I_{21}(x), \partial_{21}(x)\right) & \left(b_{22}(x), I_{22}(x), \partial_{22}(x)\right) & \cdots & \left(b_{2 h}(x), I_{2 h}(x), \partial_{2 h}(x)\right) \\
\left(b_{31}(x), I_{31}(x), \partial_{31}(x)\right) & \left(b_{32}(x), I_{32}(x), \partial_{32}(x)\right) & \cdots & \left(b_{3 h}(x), I_{3 h}(x), \partial_{3 h}(x)\right) \\
\vdots & \vdots & \ddots & \vdots \\
\left(b_{h 1}(x), I_{h 1}(x), \partial_{h 1}(x)\right) & \left(b_{h 2}(x), I_{h 2}(x), \partial_{h 2}(x)\right) & \cdots & \left(b_{h h}(x), I_{h h}(x), \partial_{h h}(x)\right)
\end{array}\right] .
$$

Step 3: if the weights of the attribute are known as a prior then use them. Otherwise, we will calculate them using the concept of neutrosophic entropy measure. Neutrosophic entropy measure is as follows:

$$
\rho_{j}=\frac{1+(1 / h) \sum_{i=1}^{h}\left(b_{i j} \log \left(b_{i j}\right)+I_{i j} \log \left(I_{i j}\right)+\partial_{i j} \log \left(\partial_{i j}\right)\right)}{\sum_{j=1}^{h}\left(1+(1 / h) \sum_{i=1}^{h} b_{i j} \log \left(b_{i j}\right)+I_{i j} \log \left(I_{i j}\right)+\partial_{i j} \log \left(\partial_{i j}\right)\right)} .
$$

Step 4: exploit the established aggregation operators to achieve the SVNN $\mathscr{F}_{t}(t=1,2, \ldots, h)$ for the alternatives $\beth_{k}$, that is, the established operators to obtained the collective overall preference values of $\mathscr{F}_{t}(t=1,2, \ldots, h)$ for the alternatives $\beth_{k}$, where $\rho=$ $\left(\rho_{1}, \rho_{2}, \ldots, \rho_{h}\right)$ is the weight vector of the attributes.

Step 5: after that, we compute the scores of all the overall values $\mathscr{F}_{t}(t=1,2, \ldots, h)$ for the alternatives $\beth_{k}$. Step 6: according to Definition 11, rank the alternatives $\beth_{k}(k=1,2, \ldots, h)$ and select the best one having the greater value.

\section{Application of Proposed Decision-Making Technique}

This section provided a numerical implementation of the problem to determining the location of the solar power plant to describe the designed DM approach.

6.1. Practical Case Study. In this segment, a case study is provided to illustrate the effectiveness and reliability of the established decision-making approach. 
The case study area was Bahawalpur District of Punjab province in Pakistan. Bahawalpur geographical coordinates are $29^{\circ} 23^{\prime} 44^{\prime \prime}$ North, $71^{\circ} 41^{\prime} 1^{\prime \prime}$ East. The Area of Bahawalpur District is $24,830 \mathrm{~km} 2$. The location of Bahawalpur is shown in Figure 1:

The required data were collected from numerous resources including governmental agencies, open sources, and related literature such as National Authority for Remote Sensing and Space Sciences, Pakistan Meteorological Authority, New and Renewable Energy Authority, Pakistan General Survey Authority, NASA POWER Prediction of Worldwide Energy Resources, United States Geological Survey, and Pakistan Environmental Affairs Agency.

Electricity plays an essential part in any nation's socioeconomic progress and social prosperity. Electricity energy should be regarded as the fundamental need for human development. In Pakistan, limited power generation is a major issue that directly restricts the country's growth. In a landmark achievement, the $100 \mathrm{MW}$ photovoltaic cells (PV) solar power project has begun commercial operations as Pakistan gradually moves to ramp up renewable energy generation in line with the global trend and to bridge the domestic shortfall. The total cost of project is \$215 million. Completed in 2015, it has a total capacity of $100 \mathrm{MW}$. Some 400,000 solar panels, spread over 200 hectares of flat desert, glare defiantly at the sun at what is known as the Quaid-eAzam Solar Power Park (QASP) in Cholistan Desert (Bahawalpur), Punjab, and named after Pakistan's founding father, Mohammad Ali Jinnah. An aerial view of Quaid-eAzam solar power park is shown in Figure 2:

The 100 MW facility is a pilot phase of a more exciting programme for the construction of the largest solar plant in the world. The location could have a capability of 5.2 million $\mathrm{Pv}$ panels generating up to $1,000 \mathrm{MW}$ of electricity once finalized in 2017, enough to power about 320,000 households. The next installation phase is already fully operational, led by Zonergy, another Chinese company.

Pakistan's National Renewable Energy Laboratories (NREL) solar power resource map has provided a major boost to the development of solar power in the open corridor regions. These regions are Pakistani Kashmir, Punjab, Sindh, and Balochistan. Here, we enlist the solar power energy project and discussed their production in Table 1:

For our research, we used a dataset comprising topographic, geological, and climatic factor. Based on several literatures, case studies concerning solar farm site selection and local conditions, different criteria were reviewed by experts, and five locations $\left\{\beth_{1}, \beth_{2}, \beth_{3}, \beth_{4}, \beth_{5}\right\}$ under five criteria were selected to evaluate the suitable sites for solar farms. The detailed criteria description is as follows:

(1) Natural factors $\left(I_{1}\right)$ : Pakistan is renowned for long hours of sunshine and powerful solar radiation. Compared to northern and southern coastal regions, central and western regions of Pakistan are exposed to greater solar intensity values. The production of solar power infrastructure has a promising future for the country. Bahawalpur is in the south of the Punjab region. Bahawalpur District solar radiation data

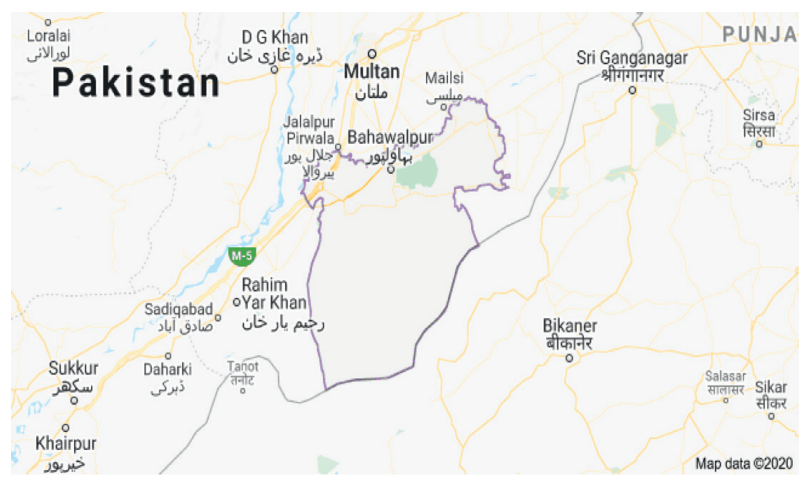

FIgURE 1: Location of Bahawalpur.

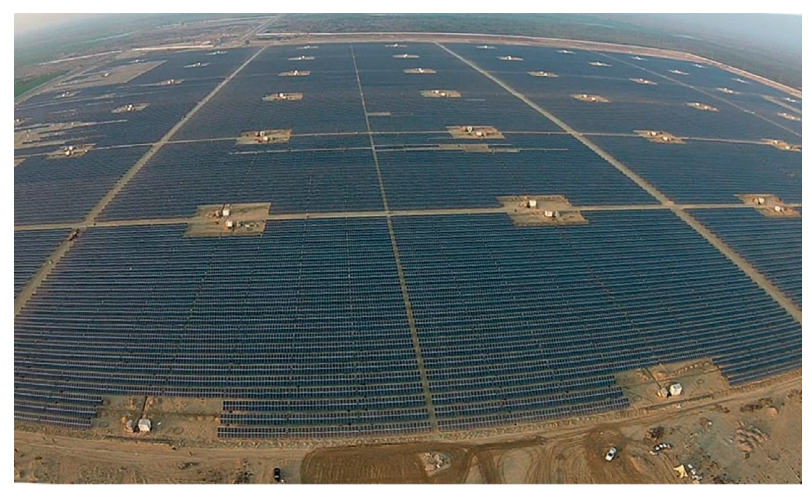

Figure 2: An aerial view of Quaid-e-Azam Solar Power Park.

acquired by Metronome software are summarized in Table 2:

In Bahawalpur District, annual average sunshine hours are 3,201 and solar radiation amount is 6,408 $\mathrm{MJ} / \mathrm{m}^{2}$.

(2) Political aspect $\left(I_{2}\right)$ : select the location that offers maximum output and minimizing project costs and gives the political point score to the government for installations of solar energy project's.

(3) Socio-economic factors $\left(I_{3}\right)$ : in order to minimize the cost of building solar farms and to reduce the cost of transporting electricity, solar farms should be located close to the existing transmission grids [59].

(4) Environmental factors $\left(I_{4}\right)$ : solar farms in areas where they negligibly interfere with existing land use outside protected areas, artificial surfaces, wetlands, aquatic areas, and forestry areas should be installed [59]. It is necessary to keep all the mechanical parts of solar park away from the water.

(5) Hydrology $\left(I_{5}\right)$ : the project site's ground water is brackish and can be reported from 7 to $8 \mathrm{~m}$ below the existing ground level. Ground water is not a means of uninterrupted fresh water availability. This region is hot and dry and receives very little rainfall. The annual average rainfall is $200-220 \mathrm{~mm}$. 
TABLE 1: Solar power projects and their production.

\begin{tabular}{lccc}
\hline Station & Location & Capacity (MW) & In service date \\
\hline Quaid-e-azam Solar Park & Bahawalpur, Punjab & 1000 & 2018 \\
OurSun Solar Power Plant & Gharo, Sindh & 50 & 18 \\
Harappa Solar Pvt. Ltd & Sahiwal, Punjab & 12 & 2018 \\
AJ Power Pvt. Ltd. & Jhelum, Punjab & 50 & 2017 \\
Gharo Solar Power Plant & Gharo, Sindh & 25 & 2017 \\
Access Electric Pvt. Ltd. & Pind Dadan Khan, Punjab, Pakistan & 2020 \\
\hline
\end{tabular}

TABLE 2: Solar radiation information of Bahawalpur.

\begin{tabular}{lcr}
\hline Months & Monthly avg. radiation quantity $\left(\mathrm{MJ} / \mathrm{m}^{2}\right)$ & Monthly average sunshine hours \\
\hline Jan & 354 & 223 \\
Feb & 441 & 224 \\
Mar & 536 & 271 \\
Apr & 638 & 288 \\
May & 686 & 308 \\
Jun & 664 & 262 \\
Jul & 651 & 276 \\
Aug & 637 & 282 \\
Sep & 606 & 285 \\
Oct & 496 & 293 \\
Nov & 386 & 263 \\
Dec & 343 & 226 \\
\hline
\end{tabular}

TABle 3: Expert evaluation information $\left(\stackrel{\circ}{D}_{1}\right)$.

\begin{tabular}{lccccc}
\hline & $I_{1}$ & $I_{2}$ & $I_{3}$ & $I_{4}$ & $I_{5}$ \\
\hline$I_{1}$ & $(0.5,0.3,0.4)$ & $(0.5,0.2,0.3)$ & $(0.2,0.2,0.6)$ & $(0.3,0.2,0.4)$ & $(0.3,0.3,0.4)$ \\
$I_{2}$ & $(0.7,0.1,0.3)$ & $(0.7,0.2,0.3)$ & $(0.6,0.3,0.2)$ & $(0.6,0.4,0.2)$ & $(0.7,0.1,0.2)$ \\
$I_{3}$ & $(0.5,0.3,0.4)$ & $(0.6,0.2,0.4)$ & $(0.6,0.1,0.2)$ & $(0.5,0.1,0.3)$ & $(0.6,0.4,0.3)$ \\
$I_{4}$ & $(0.7,0.3,0.2)$ & $(0.7,0.2,0.2)$ & $(0.4,0.5,0.2)$ & $(0.5,0.2,0.2)$ & $(0.4,0.5,0.4)$ \\
$I_{5}$ & $(0.4,0.1,0.3)$ & $(0.5,0.1,0.2)$ & $(0.4,0.1,0.5)$ & $(0.4,0.3,0.6)$ & $(0.3,0.2,0.4)$ \\
\hline
\end{tabular}

The expert panel was asked in this assessment to use SV neutrosophic information to identify the best location for solar power plant.

Step 1: the expert evaluation information using the single-valued NSs is given in Table 3.

Step 2: there is only one expert involved in this case study, so we would not need to determine the accumulated decision matrix here.

Step 3: known criteria weight vector is

$\rho=\left\{\rho_{1}=0.15, \rho_{2}=0.28, \rho_{3}=0.20, \rho_{4}=0.22, \rho_{4}=0.15\right\}$.

Step 4: evaluate the overall perfumes of the alternatives, and we utilized proposed Yager aggregation operators as shown in Tables 4 and 5 .
Step 6: compute the score value of the each collective SVNS information of each alternative as shown in Table 6:

Step 7: select the optimal alternative according the maximum score value calculated in Table 7.

We can conclude from this abovecomputational process that location $\lambda_{2}$ is the best for the installation of the solar power plant, among others, and therefore, it is highly recommended.

\section{Comparison Analysis}

We provide some appropriate examples below to test the potential and efficacy of the established decision-making approach and to compare it with the recent findings.

The use of existing methods and different aggregation operators for computed aggregate information is shown in Tables 8-10. 
TABLE 4: Yager weighted averaging.

\begin{tabular}{lccr}
\hline & SVNYWA & SVNYOWA & SVNYHWA \\
\hline$I_{1}$ & $(0.38574,0.22863,0.40300)$ & $(0.38897,0.24833,0.40881)$ & $(0.37709,0.24237,0.39733)$ \\
$I_{2}$ & $(0.65984,0.22579,0.24138)$ & $(0.66475,0.20118,0.24634)$ & $(0.65688,0.21437,0.24039)$ \\
\lrcorner$_{3}$ & $(0.56506,0.19569,0.31875)$ & $(0.55919,0.20690,0.31662)$ & $(0.56017,0.23247,0.31596)$ \\
$I_{4}$ & $(0.56718,0.30645,0.22669)$ & $(0.56559,0.32642,0.22669)$ & $(0.55677,0.29594,0.23214)$ \\
$I_{5}$ & $(0.41797,0.15495,0.37398)$ & $(0.39761,0.14876,0.37839)$ & $(0.41448,0.14641,0.38844)$ \\
\hline
\end{tabular}

TABle 5: Yager weighted geometric.

\begin{tabular}{lccr}
\hline & SVNYWG & SVNYOWG & SVNYHWG \\
\hline$\lrcorner_{1}$ & $(0.3544,0.23452,0.42473)$ & $(0.36023,0.25495,0.42367)$ & $(0.35023,0.24899,0.41376)$ \\
\lrcorner$_{2}$ & $(0.65445,0.25961,0.24799)$ & $(0.65955,0.23685,0.25298)$ & $(0.65143,0.24738,0.24698)$ \\
\lrcorner$_{3}$ & $(0.56034,0.23,0.33181)$ & $(0.55424,0.24698,0.32710)$ & $(0.55525,0.27404,0.32542)$ \\
\lrcorner$_{4}$ & $(0.53127,0.34785,0.24083)$ & $(0.52893,0.36290,0.24083)$ & $(0.52460,0.33719,0.24819)$ \\
\lrcorner$_{5}$ & $(0.40949,0.17916,0.42178)$ & $(0.39,0.16911,0.41279)$ & $(0.40687,0.16911,0.43127)$ \\
\hline
\end{tabular}

TABLE 6: Score value.

\begin{tabular}{|c|c|c|c|c|c|}
\hline & $\mathcal{S}_{\gamma}\left(\beth_{1}\right)$ & $\mathcal{S}_{\gamma}\left(\beth_{2}\right)$ & $\mathcal{S}_{\gamma}\left(\beth_{3}\right)$ & $\mathcal{S}_{\gamma}\left(\beth_{4}\right)$ & $\delta_{\gamma}\left(\beth_{5}\right)$ \\
\hline SVNYWA & -0.245899 & 0.192675 & 0.0506162 & 0.0340323 & -0.110965 \\
\hline SVNYOWA & -0.268177 & 0.217225 & 0.035665 & 0.0124742 & -0.129537 \\
\hline SVNYHWA & -0.262617 & 0.202113 & 0.0117392 & 0.0286902 & -0.120376 \\
\hline SVNYWG & -0.304856 & 0.14685 & -0.00147216 & -0.0574041 & -0.191455 \\
\hline SVNYOWG & -0.318391 & 0.169723 & -0.0198481 & -0.0747995 & -0.191911 \\
\hline SVNYHWG & -0.31253 & 0.157063 & -0.0442137 & -0.0607824 & -0.19352 \\
\hline
\end{tabular}

TABLE 7: Ranking.

\begin{tabular}{|c|c|c|}
\hline & Score ranking & Best alternative \\
\hline SVNYWA & $\delta_{\gamma}\left(I_{2}\right)>\delta_{\gamma}\left(I_{3}\right)>\mathcal{S}_{\gamma}\left(I_{4}\right)>\mathcal{S}_{\gamma}\left(I_{5}\right)>\mathcal{S}_{\gamma}\left(I_{1}\right)$ & $I_{2}$ \\
\hline SVNYOWA & $\mathcal{S}_{\gamma}\left(I_{2}\right)>\mathcal{S}_{\gamma}\left(I_{3}\right)>\mathcal{S}_{\gamma}\left(I_{4}\right)>\mathcal{S}_{\gamma}\left(I_{5}\right)>\mathcal{S}_{\gamma}\left(I_{1}\right)$ & $a_{2}$ \\
\hline SVNYHWA & $\delta_{\gamma}\left(I_{2}\right)>\delta_{\gamma}\left(I_{4}\right)>\delta_{\gamma}\left(I_{3}\right)>\delta_{\gamma}\left(I_{5}\right)>\delta_{\gamma}\left(I_{1}\right)$ & $a_{2}$ \\
\hline SVNYWG & $\mathcal{S}_{\gamma}\left(z_{2}\right)>\mathcal{S}_{\gamma}\left(z_{3}\right)>\mathcal{S}_{\gamma}\left(I_{4}\right)>\mathcal{S}_{\gamma}\left(z_{5}\right)>\mathcal{S}_{\gamma}\left(z_{1}\right)$ & $a_{2}$ \\
\hline SVNYOWG & $\mathcal{S}_{\gamma}\left(I_{2}\right)>\mathcal{S}_{\gamma}\left(I_{3}\right)>\mathcal{S}_{\gamma}\left(I_{4}\right)>\mathcal{S}_{\gamma}\left(I_{5}\right)>\mathcal{S}_{\gamma}\left(I_{1}\right)$ & $a_{2}$ \\
\hline SVNYHWG & $\mathcal{S}_{\gamma}\left(I_{2}\right)>\mathcal{S}_{\gamma}\left(I_{3}\right)>\mathcal{S}_{\gamma}\left(I_{4}\right)>\mathcal{S}_{\gamma}\left(I_{5}\right)>\mathcal{S}_{\gamma}\left(I_{1}\right)$ & $\beth_{2}$ \\
\hline
\end{tabular}

TABLE 8: Existing average aggregated SVN information.

\begin{tabular}{lcccr}
\hline & SVNWA [60] & SVNOWA [60] & NWA [55] & SVNFWA [61] \\
\hline$\lrcorner_{1}$ & $(0.37,0.22,0.40)$ & $(0.38,0.24,0.40)$ & $(0.37,0.23,0.42)$ & $(0.37,0.22,0.40)$ \\
\lrcorner$_{2}$ & $(0.66,0.20,0.23)$ & $(0.66,0.18,0.24)$ & $(0.66,0.24,0.24)$ & $(0.66,0.20,0.23)$ \\
\lrcorner$_{3}$ & $(0.56,0.17,0.31)$ & $(0.55,0.18,0.31)$ & $(0.56,0.21,0.32)$ & $(0.56,0.17,0.31)$ \\
$a_{4}$ & $(0.57,0.29,0.22)$ & $(0.57,0.31,0.22)$ & $(0.57,0.33,0.23)$ & $(0.56,0.29,0.22)$ \\
$I_{5}$ & $(0.41,0.14,0.36)$ & $(0.39,0.13,0.36)$ & $(0.41,0.16,0.41)$ & $(0.41,0.14,0.36)$ \\
\hline
\end{tabular}

TABLE 9: Existing average aggregated SVN information.

\begin{tabular}{lcccr}
\hline & SVNHWA [46] $\gamma=2$ & SVNHWA [46] $\gamma=3$ & L-SVNWA [62] & L-SVNOWA [62] \\
\hline$I_{1}$ & $(0.37,0.22,0.40)$ & $(0.36,0.22,0.40)$ & $(0.31,0.17,0.35)$ & $(0.32,0.19,0.36)$ \\
\lrcorner$_{2}$ & $(0.66,0.20,0.23)$ & $(0.66,0.20,0.23)$ & $(0.64,0.19,0.23)$ & $(0.65,0.17,0.23)$ \\
\lrcorner$_{3}$ & $(0.56,0.17,0.31)$ & $(0.56,0.18,0.31)$ & $(0.49,0.17,0.33)$ & $(0.48,0.18,0.33)$ \\
$I_{4}$ & $(0.56,0.29,0.22)$ & $(0.56,0.30,0.22)$ & $(0.55,0.27,0.19)$ & $(0.55,0.29,0.19)$ \\
$I_{5}$ & $(0.41,0.14,0.36)$ & $(0.41,0.14,0.37)$ & $(0.28,0.12,0.37)$ & $(0.24,0.12,0.38)$ \\
\hline
\end{tabular}


TABLE 10: Existing geometric aggregated SVN information.

\begin{tabular}{lccrr}
\hline & ST-SVNWA [63] & ST-SVNWG [63] & ST-SVNOWA [63] & ST-SVNOWG [63] \\
\hline$a_{1}$ & $(0.56,0.02,0.07)$ & $(0.50,0.02,0.08)$ & $(0.56,0.02,0.08)$ & $(0.51,0.03,0.08)$ \\
$a_{2}$ & $(0.86,0.02,0.02)$ & $(0.85,0.03,0.03)$ & $(0.86,0.01,0.02)$ & $(0.85,0.02,0.03)$ \\
$a_{3}$ & $(0.77,0.01,0.04)$ & $(0.76,0.02,0.05)$ & $(0.77,0.01,0.04)$ & $(0.76,0.03,0.05)$ \\
$a_{4}$ & $(0.78,0.04,0.02)$ & $(0.73,0.06,0.02)$ & $(0.78,0.04,0.02)$ & $(0.72,0.06,0.02)$ \\
$a_{5}$ & $(0.60,0.09,0.06)$ & $(0.59,0.01,0.08)$ & $(0.58,0.09,0.06)$ & $(0.57,0.01,0.08)$ \\
\hline
\end{tabular}

TABLE 11: Overall ranking of the alternatives.

\begin{tabular}{|c|c|c|}
\hline Existing operators & Ranking & Best alternative \\
\hline NWA [55] & $\mathcal{S}_{\gamma}\left(I_{2}\right)>\mathcal{S}_{\gamma}\left(\beth_{3}\right)>\mathcal{S}_{\gamma}\left(\beth_{1}\right)>\mathcal{S}_{\gamma}\left(I_{5}\right)>\mathcal{S}_{\gamma}\left(I_{4}\right)$ & $I_{2}$ \\
\hline SVNWA [60] & $\mathcal{S}_{\gamma}\left(I_{2}\right)>\mathcal{S}_{\gamma}\left(\beth_{3}\right)>\mathcal{S}_{\gamma}\left(I_{1}\right)>\mathcal{S}_{\gamma}\left(I_{5}\right)>\mathcal{S}_{\gamma}\left(I_{4}\right)$ & $I_{2}$ \\
\hline SVNOWA [60] & $\mathcal{S}_{\gamma}\left(I_{2}\right)>\mathcal{S}_{\gamma}\left(\beth_{3}\right)>\mathcal{S}_{\gamma}\left(\beth_{1}\right)>\mathcal{S}_{\gamma}\left(I_{5}\right)>\mathcal{S}_{\gamma}\left(I_{4}\right)$ & $a_{2}$ \\
\hline SVNFWA [61] & $\mathcal{S}_{\gamma}\left(\beth_{2}\right)>\mathcal{S}_{\gamma}\left(\beth_{3}\right)>\mathcal{S}_{\gamma}\left(\beth_{1}\right)>\mathcal{S}_{\gamma}\left(\beth_{5}\right)>\mathcal{S}_{\gamma}\left(\beth_{4}\right)$ & $I_{2}$ \\
\hline SVNHWA [46] $\gamma=2$ & 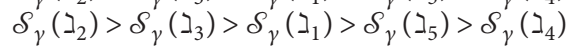 & $I_{2}$ \\
\hline SVNHWA [46] $\gamma=3$ & $\delta_{\gamma}\left(z_{2}\right)>\delta_{\gamma}\left(z_{3}\right)>\delta_{\gamma}\left(I_{1}\right)>\mathcal{S}_{\gamma}\left(I_{5}\right)>\mathcal{S}_{\gamma}\left(z_{4}\right)$ & $\lambda_{2}$ \\
\hline L-SVNWA [62] & $\delta_{\gamma}\left(I_{2}\right)>\delta_{\gamma}\left(\beth_{3}\right)>\delta_{\gamma}\left(I_{1}\right)>\delta_{\gamma}\left(I_{5}\right)>\delta_{\gamma}\left(I_{4}\right)$ & $a_{2}$ \\
\hline L-SVNOWA [62] & $\mathcal{S}_{\gamma}\left(z_{2}\right)>\delta_{\gamma}\left(z_{3}\right)>\mathcal{S}_{\gamma}\left(\beth_{1}\right)>\mathcal{S}_{\gamma}\left(z_{5}\right)>\mathcal{S}_{\gamma}\left(z_{4}\right)$ & $\beth_{2}$ \\
\hline L-SVNWG [62] & $\delta_{\gamma}\left(z_{2}\right)>\delta_{\gamma}\left(z_{3}\right)>\mathcal{S}_{\gamma}\left(z_{1}\right)>\mathcal{S}_{\gamma}\left(I_{5}\right)>\mathcal{S}_{\gamma}\left(z_{4}\right)$ & $I_{2}$ \\
\hline ST-SVNWA [63] & $\mathcal{S}_{\gamma}\left(I_{2}\right)>\mathcal{S}_{\gamma}\left(\beth_{3}\right)>\mathcal{S}_{\gamma}\left(I_{1}\right)>\mathcal{S}_{\gamma}\left(I_{5}\right)>\mathcal{S}_{\gamma}\left(I_{4}\right)$ & $\beth_{2}$ \\
\hline ST-SVNWG [63] & $\delta_{\gamma}\left(z_{2}\right)>\delta_{\gamma}\left(I_{3}\right)>\mathcal{S}_{\gamma}\left(I_{1}\right)>\mathcal{S}_{\gamma}\left(I_{5}\right)>\mathcal{S}_{\gamma}\left(z_{4}\right)$ & $I_{2}$ \\
\hline ST-SVNOWA [63] & $\delta_{\gamma}\left(z_{2}\right)>\delta_{\gamma}\left(z_{3}\right)>\mathcal{S}_{\gamma}\left(I_{1}\right)>\mathcal{S}_{\gamma}\left(I_{5}\right)>\mathcal{S}_{\gamma}\left(z_{4}\right)$ & $I_{2}$ \\
\hline ST-SVNOWG [63] & $\mathcal{S}_{\gamma}\left(I_{2}\right)>\mathcal{S}_{\gamma}\left(I_{3}\right)>\mathcal{S}_{\gamma}\left(I_{1}\right)>\mathcal{S}_{\gamma}\left(I_{5}\right)>\mathcal{S}_{\gamma}\left(I_{4}\right)$ & $I_{2}$ \\
\hline
\end{tabular}

TABLE 12: Overall ranking of the alternatives.

\begin{tabular}{lll}
\hline Proposed operators & \multicolumn{1}{c}{ Ranking } & Best alternative \\
\hline SVNYWA & $\mathcal{S}_{\gamma}\left(\beth_{2}\right)>\mathcal{S}_{\gamma}\left(\beth_{3}\right)>\mathcal{S}_{\gamma}\left(\beth_{4}\right)>\mathcal{S}_{\gamma}\left(\beth_{5}\right)>\mathcal{S}_{\gamma}\left(\beth_{1}\right)$ & $\beth_{2}$ \\
SVNYOWA & $\mathcal{S}_{\gamma}\left(\beth_{2}\right)>\mathcal{S}_{\gamma}\left(\beth_{3}\right)>\mathcal{S}_{\gamma}\left(\beth_{4}\right)>\mathcal{S}_{\gamma}\left(\beth_{5}\right)>\mathcal{S}_{\gamma}\left(\beth_{1}\right)$ & $\beth_{2}$ \\
SVNYHWA & $\mathcal{S}_{\gamma}\left(\beth_{2}\right)>\mathcal{S}_{\gamma}\left(\beth_{4}\right)>\mathcal{S}_{\gamma}\left(\beth_{3}\right)>\mathcal{S}_{\gamma}\left(\beth_{5}\right)>\mathcal{S}_{\gamma}\left(\beth_{1}\right)$ & $\beth_{2}$ \\
SVNYWG & $\mathcal{S}_{\gamma}\left(\beth_{2}\right)>\mathcal{S}_{\gamma}\left(\beth_{3}\right)>\mathcal{S}_{\gamma}\left(\beth_{4}\right)>\mathcal{S}_{\gamma}\left(\beth_{5}\right)>\mathcal{S}_{\gamma}\left(\beth_{1}\right)$ & $\beth_{2}$ \\
SVNYOWG & $\mathcal{S}_{\gamma}\left(\beth_{2}\right)>\mathcal{S}_{\gamma}\left(\beth_{3}\right)>\mathcal{S}_{\gamma}\left(\beth_{4}\right)>\mathcal{S}_{\gamma}\left(\beth_{5}\right)>\mathcal{S}_{\gamma}\left(\beth_{1}\right)$ & $\beth_{2}$ \\
SVNYHWG & $\mathcal{S}_{\gamma}\left(\beth_{2}\right)>\mathcal{S}_{\gamma}\left(\beth_{3}\right)>\mathcal{S}_{\gamma}\left(\beth_{4}\right)>\mathcal{S}_{\gamma}\left(\beth_{5}\right)>\mathcal{S}_{\gamma}\left(\beth_{1}\right)$ & \\
\hline
\end{tabular}

Now, according to collective data, the overall ranking of alternative is as shown in Tables 11 and 12 .

From the findings of the proposed operators and the existing methods, we conclude that the ranking lists are the same. The generalized and novel approach to address uncertainty in DM problems is the Yager operators under the SVNS environment. Yager norm-based aggregation operators under single-valued NS environment are more flexible and efficient in assessing the best alternative in real-world problems.

\section{Conclusion}

Single-valued NS is a general extension of intuitionistic FS, picture FS, which is more capable of dealing with incomplete and inconsistent information. Therefore, it is widely used in various fields. Single-valued NS tackles the vagueness and uncertain information in real-world complex problems with a more flexible and effective way. In addition, the Yager norms have a more generalized framework that works effectively to incorporate complex information. We are motivated by the deficiencies of the existing methods and the beneficial features of the Yager AgOs to work towards improving a successful merger with SVNNs.

In this study, under the single-valued NS model, we modified the multiskilled Yager AgOs to integrate the benefits and flexibility of both theories. Later, we explore operational laws of SVNNs to construct single-valued NS AgOs that comply with the principles of Yager operations. We have established the single-valued neutrosophic weighted averaging, ordered weighted averaging, hybrid weighted averaging, weighted geometric, ordered weighted geometric, and hybrid weighted geometric aggregation operators to aggregate the SVNNs. Some of the main characteristics of the proposed operators have been studied, including idempotency, boundedness, and monotonicity. The main objective of this study is to present a strategy to address MAGDM that includes single-valued NS evaluations based on the proposed operators. The theoretical basis of AgOs needs to be carefully considered in preparation for their use in MAGDM. A practical example is provided to demonstrate the implementation of the established strategy for the selection of a suitable location for solar power stations. The comparison analysis of our proposed theory was 
conducted with the existing operators. The superiority of our proposed operators over the existing DM method has been highlighted. We examined the effect of different parameter values on the results of MAGDM issues. In short, this article creates a tool that has the rich properties of Yager AgOs and the single-valued NS model's flexibility. We will expand our models to single-valued NS hesitant fuzzy set environments in future research.

\section{Data Availability}

No data were used to support this study.

\section{Ethical Approval}

This article does not contain any studies with human participants or animals performed by any of the authors.

\section{Conflicts of Interest}

The authors declare that they have no conflicts of interest.

\section{References}

[1] L. A. Zadeh, "Fuzzy sets," Information and Control, vol. 8, no. 3, pp. 338-353, 1965.

[2] K. Attanassov, "Intuitionistic fuzzy sets," Fuzzy Sets and Systems, vol. 20, pp. 87-96, 1986.

[3] Z. Ali and T. Mahmood, "Complex neutrosophic generalised dice similarity measures and their application to decision making," CAAI Transactions on Intelligence Technology, vol. 5, no. 2, p. $78,2020$.

[4] C. Huang, M. Lin, and Z. Xu, "Pythagorean fuzzy MULTIMOORA method based on distance measure and score function: its application in multicriteria decision making process," Knowledge and Information Systems, vol. 62, no. 11, pp. 4373-4406, 2020.

[5] D.-F. Li, T. Mahmood, Z. Ali, and Y. Dong, "Decision making based on interval-valued complex single-valued neutrosophic hesitant fuzzy generalized hybrid weighted averaging operators," Journal of Intelligent \& Fuzzy Systems, vol. 38, no. 4, pp. 4359-4401, 2020.

[6] M. Lin, C. Huang, and Z. Xu, "MULTIMOORA based MCDM model for site selection of car sharing station under picture fuzzy environment," Sustainable Cities and Society, vol. 53, p. $101873,2020$.

[7] M. Lin, X. Li, and L. Chen, "Linguistic q -rung orthopair fuzzy sets and their interactional partitioned Heronian mean aggregation operators," International Journal of Intelligent Systems, vol. 35, no. 2, pp. 217-249, 2020.

[8] M. Lin, Z. Chen, H. Liao, and Z. Xu, "ELECTRE II method to deal with probabilistic linguistic term sets and its application to edge computing," Nonlinear Dynamics, vol. 96, no. 3, pp. 2125-2143, 2019.

[9] L. Wang and N. Li, "Pythagorean fuzzy interaction power Bonferroni mean aggregation operators in multiple attribute decision making," International Journal of Intelligent Systems, vol. 35, no. 1, pp. 150-183, 2020.

[10] L. Wang, H. Garg, and N. Li, "Pythagorean fuzzy interactive Hamacher power aggregation operators for assessment of express service quality with entropy weight," Soft Computing, pp. 1-21, 2020.
[11] Z. Xu and R. R. Yager, "Some geometric aggregation operators based on intuitionistic fuzzy sets," International Journal of General Systems, vol. 35, no. 4, pp. 417-433, 2006.

[12] Z. Xu, "Intuitionistic fuzzy aggregation operators," IEEE Transactions on Fuzzy Systems, vol. 15, no. 6, pp. 1179-1187, 2007.

[13] R. R. Yager, "Pythagorean membership grades in multicriteria decision making," IEEE Transactions on Fuzzy Systems, vol. 22, no. 4, pp. 958-965, 2013.

[14] L. H. Son, "Generalized picture distance measure and applications to picture fuzzy clustering," Applied Soft Computing, vol. 46, no. C, pp. 284-295, 2016.

[15] B. C. Cuong, Picture Fuzzy Sets-First Results. Part 1, Seminar Neuro-Fuzzy Systems with Applications, Institute of Mathematics, Hanoi, Vietnam, 2013.

[16] B. C. Cuong and V. Kreinovich, "Picture fuzzy sets," Journal of Computer Science and Cybernetics, vol. 30, no. 4, pp. 409-420, 2014.

[17] G. Wei, "Picture fuzzy aggregation operators and their application to multiple attribute decision making," Journal of Intelligent \& Fuzzy Systems, vol. 33, no. 2, pp. 713-724, 2017.

[18] S. Ashraf, T. Mahmood, S. Abdullah, and Q. Khan, "Different approaches to multi-criteria group decision making problems for picture fuzzy environment," Bulletin of the Brazilian Mathematical Society, New Series, vol. 50, no. 2, pp. 373-397, 2019.

[19] M. Khan, P. Kumam, S. Ashraf, and W. Kumam, "Generalized picture fuzzy soft sets and their application in decision support systems," Symmetry, vol. 11, no. 3, p. 415, 2019.

[20] S. Khan, S. Abdullah, L. Abdullah, and S. Ashraf, "Logarithmic aggregation operators of picture fuzzy numbers for multiattribute decision making problems," Mathematics, vol. 7, no. 7, p. 608, 2019.

[21] M. Qiyas, S. Abdullah, S. Ashraf, and M. Aslam, "Utilizing linguistic picture fuzzy aggregation operators for multipleattribute decision-making problems," International Journal of Fuzzy Systems, vol. 22, no. 1, pp. 310-320, 2020.

[22] S. Ashraf, S. Abdullah, T. Mahmood, and M. Aslam, "Cleaner production evaluation in gold mines using novel distance measure method with cubic picture fuzzy numbers," International Journal of Fuzzy Systems, vol. 21, no. 8, pp. 24482461, 2019.

[23] M. Qiyas, S. Abdullah, S. Ashraf, and L. Abdullah, "Linguistic picture fuzzy Dombi aggregation operators and their application in multiple attribute group decision making problem," Mathematics, vol. 7, no. 8, p. 764, 2019.

[24] S. Ashraf, S. Abdullah, and F. Muneeza, "Some novel aggregation operators for cubic picture fuzzy information: application in multi-attribute decision support problem," Granular Computing, pp. 1-16, 2020.

[25] S. Ashraf, S. Abdullah, T. Mahmood, F. Ghani, and T. Mahmood, "Spherical fuzzy sets and their applications in multi-attribute decision making problems," Journal of Intelligent \& Fuzzy Systems, vol. 36, no. 3, pp. 2829-2844, 2019.

[26] S. Ashraf and S. Abdullah, "Spherical aggregation operators and their application in multiattribute group decision-making," International Journal of Intelligent Systems, vol. 34, no. 3, pp. 493-523, 2019.

[27] S. Ashraf, S. Abdullah, M. Aslam, M. Qiyas, and M. A. Kutbi, "Spherical fuzzy sets and its representation of spherical fuzzy t-norms and t-conorms," Journal of Intelligent \& Fuzzy Systems, vol. 36, no. 6, pp. 6089-6102, 2019.

[28] H. Jin, S. Ashraf, S. Abdullah, M. Qiyas, M. Bano, and S. Zeng, "Linguistic spherical fuzzy aggregation operators and their 
applications in multi-attribute decision making problems," Mathematics, vol. 7, no. 5, p. 413, 2019.

[29] S. Ashraf, S. Abdullah, and T. Mahmood, "Spherical fuzzy Dombi aggregation operators and their application in group decision making problems," Journal of Ambient Intelligence and Humanized Computing, vol. 11, no. 7, pp. 2731-2749, 2019.

[30] S. Ashraf, S. Abdullah, and T. Mahmood, "GRA method based on spherical linguistic fuzzy Choquet integral environment and its application in multi-attribute decision-making problems," Mathematical Sciences, vol. 12, no. 4, pp. 263-275, 2018.

[31] M. Rafiq, S. Ashraf, S. Abdullah, T. Mahmood, and S. Muhammad, "The cosine similarity measures of spherical fuzzy sets and their applications in decision making," Journal of Intelligent \& Fuzzy Systems, vol. 36, no. 6, pp. 6059-6073, 2019.

[32] S. Ashraf, S. Abdullah, and L. Abdullah, "Child development influence environmental factors determined using spherical fuzzy distance measures," Mathematics, vol. 7, no. 8, p. 661, 2019.

[33] S. Zeng, A. Hussain, T. Mahmood, M. Irfan Ali, S. Ashraf, and M. Munir, "Covering-based spherical fuzzy rough set model hybrid with TOPSIS for multi-attribute decision-making," Symmetry, vol. 11, no. 4, p. 547, 2019.

[34] F. K. Gündoğdu and C. Kahraman, "A novel fuzzy TOPSIS method using emerging interval-valued spherical fuzzy sets," Engineering Applications of Artificial Intelligence, vol. 85, pp. 307-323, 2019.

[35] S. Ashraf and S. Abdullah, "Emergency decision support modeling for COVID-19 based on spherical fuzzy information," International Journal of Intelligent Systems, vol. 35, no. 11, p. 1601, 2020.

[36] S. Ashraf, S. Abdullah, and M. Aslam, "Symmetric sum based aggregation operators for spherical fuzzy information: application in multi-attribute group decision making problem," Journal of Intelligent \& Fuzzy Systems, vol. 38, no. 4, pp. 5241-5255, 2020.

[37] F. K. Gundogdu and C. Kahraman, "Extension of WASPAS with spherical fuzzy sets," Informatica, vol. 30, no. 2, pp. 269-292, 2019.

[38] Y. Jin, S. Ashraf, and S. Abdullah, "Spherical fuzzy logarithmic aggregation operators based on entropy and their application in decision support systems," Entropy, vol. 21, no. 7, p. 628, 2019.

[39] S. A. S. Shishavan, F. Kutlu Gündoğdu, E. Farrokhizadeh, Y. Donyatalab, and C. Kahraman, "Novel similarity measures in spherical fuzzy environment and their applications," Engineering Applications of Artificial Intelligence, vol. 94, p. 103837, 2020.

[40] F. K. Gündoğdu and C. Kahraman, "A novel spherical fuzzy analytic hierarchy process and its renewable energy application," Soft Computing, vol. 24, no. 6, pp. 4607-4621, 2020.

[41] F. K. Gündoğdu and C. Kahraman, "A novel spherical fuzzy QFD method and its application to the linear delta robot technology development," Engineering Applications of Artificial Intelligence, vol. 87, Article ID 103348, 2020.

[42] F. Smarandache, A Unifying Field in Logics Neutrosophy: Neutrosophic Probability, Set and Logic, American Research Press, Santa Fe, NM, USA, 1998.

[43] J. Ye, "A multicriteria decision-making method using aggregation operators for simplified neutrosophic sets," Journal of Intelligent \& Fuzzy Systems, vol. 26, no. 5, pp. 2459-2466, 2014.
[44] J.-j. Peng, J.-q. Wang, X.-h. Wu, J. Wang, and X.-h. Chen, "Multivalued neutrosophic sets and power aggregation operators with their applications in multi-criteria group decision-making problems," International Journal of Computational Intelligence Systems, vol. 8, no. 2, pp. 345-363, 2015.

[45] J. Chen and J. Ye, "Some single-valued neutrosophic Dombi weighted aggregation operators for multiple attribute decision-making," Symmetry, vol. 9, no. 6, p. 82, 2017.

[46] P. Liu, Y. Chu, Y. Li, and Y. Chen, "Some generalized neutrosophic number Hamacher aggregation operators and their application to group decision making," International Journal of Fuzzy Systems, vol. 16, no. 2, 2014.

[47] G. Wei and Z. Zhang, "Some single-valued neutrosophic Bonferroni power aggregation operators in multiple attribute decision making," Journal of Ambient Intelligence and $\mathrm{Hu}$ manized Computing, vol. 10, no. 3, pp. 863-882, 2019.

[48] P. Liu, T. Mahmood, and Q. Khan, "Group decision making based on power Heronian aggregation operators under linguistic neutrosophic environment," International Journal of Fuzzy Systems, vol. 20, no. 3, pp. 970-985, 2018.

[49] H. Garg and C. Nancy, "Linguistic single-valued neutrosophic prioritized aggregation operators and their applications to multiple-attribute group decision-making," Journal of Ambient Intelligence and Humanized Computing, vol. 9, no. 6, pp. 1975-1997, 2018.

[50] M. Akram and G. Shahzadi, "A hybrid decision-making model under q-rung orthopair fuzzy Yager aggregation operators," Granular Computing, pp. 1-15, 2020.

[51] M. Akram, X. Peng, and A. Sattar, "Multi-criteria decision-making model using complex pythagorean fuzzy yager aggregation operators," Arabian Journal for Science and Engineering, pp. 1-27, 2020.

[52] G. Shahzadi, M. Akram, and A. N. Al-Kenani, "decision-making approach under Pythagorean fuzzy Yager weighted operators," Mathematics, vol. 8, no. 1, p. 70, 2020.

[53] H. Garg, G. Shahzadi, and M. Akram, "Decision-making analysis based on fermatean fuzzy yager aggregation operators with application in COVID-19 testing facility," Mathematical Problems in Engineering, vol. 2020, p. 1, Article ID 7279027, 2020.

[54] H. Wang, F. Smarandache, Y. Zhang, and R. Sunderraman, "Single valued neutrosophic sets," Multispace Multistructure, vol. 4, pp. 410-413, 2010.

[55] J. Ye, "Subtraction and division operations of simplified neutrosophic sets," Information, vol. 8, no. 2, p. 51, 2017.

[56] X. Zhang, C. Bo, F. Smarandache, and J. Dai, "New inclusion relation of neutrosophic sets with applications and related lattice structure," International Journal of Machine Learning and Cybernetics, vol. 9, no. 10, pp. 1753-1763, 2018.

[57] J. Ye, "Single valued neutrosophic cross-entropy for multicriteria decision making problems," Applied Mathematical Modelling, vol. 38, no. 3, pp. 1170-1175, 2014.

[58] R. R. Yager, "Aggregation operators and fuzzy systems modeling," Fuzzy Sets and Systems, vol. 67, no. 2, pp. 129-145, 1994.

[59] A. Khaliq, A. Ikram, and M. Salman, "June. Quaid-e-Azam solar power park: prospects and challenges," in Power Generation System and Renewable Energy Technologies (PGSRET), pp. 1-6, IEEE, 2015.

[60] J.-J. Peng, J.-Q. Wang, J. Wang, H.-Y. Zhang, and X.-H. Chen, "Simplified neutrosophic sets and their applications in multicriteria group decision-making problems," International Journal of Systems Science, vol. 47, no. 10, pp. 2342-2358, 2016. 
[61] Nancy and H. Garg, "Novel single-valued neutrosophic aggregated operators under Frank norm operation and its application to decision-making process," International Journal for Uncertainty Quantification, vol. 6, pp. 361-375, 2016.

[62] H. Garg and F. Nancy, "New logarithmic operational laws and their applications to multiattribute decision making for singlevalued neutrosophic numbers," Cognitive Systems Research, vol. 52, pp. 931-946, 2018.

[63] S. Ashraf, S. Abdullah, S. Zeng, H. Jin, and F. Ghani, "Fuzzy decision support modeling for hydrogen power plant selection based on single valued neutrosophic sine trigonometric aggregation operators," Symmetry, vol. 12, no. 2, p. 298, 2020. 